\title{
The Physics of Heavy Quark Distributions in Hadrons: Collider Tests
}

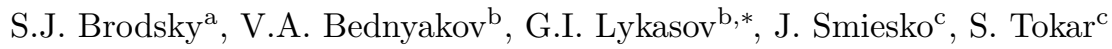 \\ ${ }^{a}$ SLAC National Accelerator Laboratory, Stanford University, Menlo Park, CA 94025, \\ United States \\ ${ }^{b}$ Joint Institute for Nuclear Research, Dubna 141980, Moscow region, Russia \\ ${ }^{c}$ Comenius University in Bratislava, Faculty of Mathematics, Physics and Informatics, \\ Mlynska Dolina, 84248 Bratislava, Slovakia
}

\begin{abstract}
We present a review of the current understanding of the heavy quark distributions in the nucleon and their impact on collider physics. The origin of strange, charm and bottom quark pairs at high light-front (LF) momentum fractions in hadron wavefunctions - the "intrinsic" quarks, is reviewed. The determination of heavy-quark parton distribution functions (PDFs) is particularly significant for the analysis of hard processes at LHC energies. We show that a careful study of the inclusive production of open charm and the production of $\gamma / Z / W$ particles, accompanied by the heavy jets at large transverse momenta can give essential information on the intrinsic heavy quark (IQ) distributions. We also focus on the theoretical predictions concerning other observables which are very sensitive to the intrinsic charm contribution to PDFs including Higgs production at high $x_{\mathrm{F}}$ and novel fixed target measurements which can be tested at the
\end{abstract} LHC.

Keywords: Quarks, gluons, charm, QCD, PDF.

\footnotetext{
*Corresponding author

Email address: lykasov@jinr.ru (G.I. Lykasov)
}

Preprint submitted to Progress in Particle and Nuclear Physics

December 1, 2016

(C) 2016. This manuscript version is made available under the Elsevier user license http://www.elsevier.com/open-access/userlicense/1.0/ 


\section{Contents}

1 Introduction 3

1.1 Motivation of this review $\ldots \ldots \ldots \ldots \ldots$

$1.2 \quad$ Outline of this Review $\ldots \ldots \ldots \ldots \ldots$

\begin{tabular}{|lll}
2 & Extrinsic and intrinsic quark components in nucleon & 8
\end{tabular}

2.1 Intrinsic charm density in a proton as a function of IC probability $w 16$

3 Intrinsic heavy quarks and hard $p p$ collisions 20

3.1 Where can one look for the intrinsic heavy quarks? . . . . . . . . 20

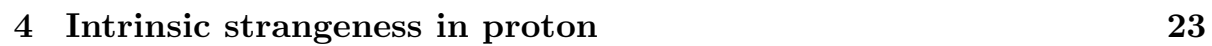

5 Global analysis of PDFs with intrinsic charm 26

\begin{tabular}{|lll}
6 & Intrinsic heavy quark signal in processes at collider energies & 27
\end{tabular}

6.1 Inclusive production of charmed meson . . . . . . . . . . . 27

6.2 Inclusive production of Higgs boson and intrinsic charm and bottom in proton . . . . . . . . . . . . . . . . . . . . . . 30

6.3 Production of prompt photon and $c$ or $b$-jet in hard $p p$ collisions 31

6.4 Production of $\gamma(Z)+c(b)$-jet within the $k_{\mathrm{T}}$-factorization and the

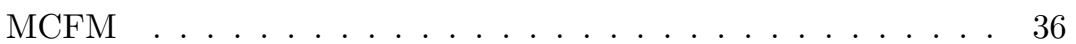

6.5 Prompt photon and open charm production . . . . . . . . . . 48

$6.6 \quad W(Z)$-boson and $b(c)$-jet production $\ldots \ldots \ldots \ldots \ldots$

\begin{tabular}{lll}
\hline 7 & Conclusion & 62
\end{tabular}

\begin{tabular}{|ll|}
\hline 8 & Acknowledgements
\end{tabular}

$\begin{array}{lll}9 & \text { References } & 65\end{array}$ 


\section{Introduction}

\subsection{Motivation of this review}

The knowledge of the heavy quark distributions of the proton can provide fundamental information on nucleon structure. Since many hard processes within the Standard Model (SM) and beyond, such as the production of heavy jets and Higgs boson production, etc., are sensitive to the heavy quark content of the nucleon, heavy quark distributions play an increasingly significant role in the physics program of the Large Hadron Collider (LHC). The parton distribution functions (PDFs) of the strange, charm, and bottom quarks and their evolution are essential inputs for the calculation of these processes within perturbative quantum chromodynamics (pQCD). The heavy quark PDFs reflect both the initial conditions at momentum transfers below a factorization scale $Q^{2}<Q_{0}^{2}$ as dictated by the non-perturbative QCD color-confining dynamics of the proton plus the effects of pQCD which generates heavy quarks from the evolution of the light quark and gluon PDFs. A global QCD analysis allows one to extract the PDFs from the comparison of hard-scattering data based on the factorization properties of QCD.

In fact, QCD predicts two separate and distinct contributions to the heavy 20 quark distributions $q\left(x, Q^{2}\right)$ of the nucleons at low and high $x$. Here $x=\frac{k^{+}}{P^{+}}=$ $\frac{k^{0}+k^{3}}{P^{0}+P^{3}}$ is the frame-independent light-front (LF) momentum fraction carried by the heavy quark in a hadron with momentum $P^{\mu}$. In the case of deep inelastic lepton-proton scattering, the LF momentum fraction variable $x$ in the proton structure functions can be identified with the Bjorken variable $x_{\mathrm{Bj}}=$

${ }_{25} \frac{Q^{2}}{2 p \cdot q}$. At small $x$, heavy-quark pairs are dominantly produced via gluon-splitting subprocess $g \rightarrow Q \bar{Q}$. The existence of the heavy quarks in the nucleon from this standard contribution is a result of the QCD evolution of the light quark and gluon PDFs. The gluon splitting contribution to the heavy-quark degrees of freedom are perturbatively calculable using the Dokshitzer-Gribov-Lipatov- 
Altarelli-Parisi (DGLAP) evolution equation [1]3. To first approximation, the heavy quark distribution falls as $(1-x)$ times the gluon distribution.

However, the QCD also predicts additional Fock state contributions to the proton structure at high $x$, such as $|u u d Q \bar{Q}\rangle$, where the heavy quark pair is multiply connected to two or more valence quarks of the proton. This contribu35 tion depends on non-perturbative distribution of valence quarks in the hadron, and is maximal at minimal off-shellness; i.e., when the constituents all have the same rapidity $y_{i}$ and thus $x_{i} \propto \sqrt{m_{i}^{2}+\vec{k}_{T i}^{2}}$. The heavy quark contributions to the nucleon's PDF thus peak at large $x$. Since they depend on the correlations determined by the valence quark distributions, these heavy quark contributions 40 are intrinsic contributions to the hadron's fundamental structure. Furthermore, since all of the intrinsic quarks in the $|u u d Q \bar{Q}\rangle$ Fock state have similar rapidities they can reinteract, leading to significant $Q$ vs. $\bar{Q}$ asymmetries. In contrast, the contribution to the heavy quark PDFs arising from gluon splitting are symmetric in $Q$ vs. $\bar{Q}$. Since they only depend on the gluon distribution, the contributions 45 generated by DGLAP evolution can be considered as extrinsic contributions.

The PDFs at a fundamental level are computed from the squares of the hadrons' light-front wavefunctions, the frame-independent eigensolutions of the QCD Light-Front Hamiltonian. The intrinsic contributions are associated with amplitudes such as $g g \rightarrow Q \bar{Q} \rightarrow g g$ in the self-energy of the proton, the analogs 50 of light-by-light scattering $\gamma \gamma \rightarrow \ell \bar{\ell} \rightarrow \gamma \gamma$ in QED, i.e., twist-6 contributions proportional to the gluon field strength of the order 3 in the operator product expansion (OPE). Thus the OPE provides a first-principle derivation for the existence of intrinsic heavy quarks.

Unlike the conventional $\log m_{\mathrm{Q}}^{2}$ dependence of the low $x$ extrinsic gluon55 splitting contribution, the probabilities for the intrinsic heavy quark Fock states at high $x$ scale as $\frac{1}{m_{\mathrm{Q}}^{2}}$ in non-Abelian QCD. In contrast the probability for a higher Fock state in an atom such as $\left|e^{+} e^{-} \ell \bar{\ell}\right\rangle$ in positronium scales as $\frac{1}{m_{\ell}^{4}}$ in Abelian QED, corresponding to the twist-8 Euler-Heisenberg light-by-light insertion. Detailed derivations based on the OPE have been given in [4, 5].

When a proton collides with another proton or nucleus, the off-shell intrinsic 
heavy quark Fock state fluctuations such as $|u u d c \bar{c}\rangle$ are materialized and can produce open or hidden charm states at high momentum fraction. For example, the comoving $u d c$ quarks in a Fock state such as $|u u d c \bar{c}\rangle$ can coalesce to produce a $\Lambda_{\mathrm{c}}(u d c)$ baryon with a high Feynman momentum fraction $x_{\mathrm{F}}=x_{\mathrm{c}}+x_{\mathrm{u}}+x_{\mathrm{d}}$ 65 or produce a $J / \psi$ with $x_{\mathrm{F}}=c \bar{c}$. Such high $x_{\mathrm{F}}$ heavy hadron events have been observed and measured with substantial cross sections at the ISR protonproton collider at fixed target experiments such as NA3 at CERN and SELEX at FermiLab. The $\Lambda_{\mathrm{b}}(u d b)$ baryon was first observed at the ISR in forward $p p \rightarrow \Lambda_{\mathrm{b}} X$ reactions at high $x_{\mathrm{F}}$.

The hypothesis of the intrinsic quark components in the proton was suggested in [6] to explain the large cross-section for the forward open charm production in $p p$ collision at ISR energies [7-10]. The magnitude of the ISR cross sections suggests that the intrinsic charm probability in the proton is approximately of order $1 \%$; however, theoretical and experimental uncertainties make it difficult to make an accurate estimate.

Intrinsic charm is also important for charm production in cosmic ray experiments that measure charm production from high energy experiments interacting in the earth's atmosphere. It also is important for estimating the high energy flux of neutrinos observed in the IceCube experiment. In fact one finds [11 that 80 the prompt neutrino flux arising from charm hadroproduction by protons interacting in the earth's atmosphere which is due to intrinsic charm is comparable to the extrinsic contribution if one normalizes the intrinsic charm differential cross sections to the ISR and the LEBC-MPS collaboration data.

Most measurements of $c$ and $b$-jet production in deep inelastic lepton-proton scattering are consistent with the extrinsic gluon-splitting perturbative origin of heavy flavor quarks [12] alone. However, these experiments are not sensitive to the heavy quark distributions at large $x$ region, where the theory predicts a non zero contribution of the so called intrinsic heavy-quark components in the proton wave function [4, 6, 13, as is suggested in Refs. 14 16.

90

The first direct experimental indication for the intrinsic heavy quarks in a nucleon was observed at the EMC measurement of the charm structure function 
at large $x$ [17. The measurement of the charm structure function at high $x_{\mathrm{Bj}}$ by the EMC experiment at CERN using deep inelastic muon-nucleus scattering showed a significant contribution to the proton structure function at large $x_{\mathrm{Bj}}$ 17. In fact, the charm structure function $c(x, Q)$ measured by the EMC collaboration was approximately 30 times higher than expected from gluon splitting and at $x_{\mathrm{Bj}}=0.42$ and $Q^{2}=75(\mathrm{GeV} / \mathrm{c})^{2}$. As shown in Refs. [18 20, the inclusion of both the HERA data and the EMC data to the global NNPDF analysis allows one to do a model independent determination of the charm content of the proton. The results of Ref. [18] suggest the perturbative origin of the charm $\mathrm{PDF}$ at $x<0.1$, which vanishes at $\mu \simeq 1.5 \mathrm{GeV} / \mathrm{c}$, but it also indicate presence of a large $x$ intrinsic component picked at $x \sim 0.5$ and carried $0.7 \pm 0.3 \%$ of the nucleon momentum with the $68 \% \mathrm{CL}$ at low scale $\mu_{0}=1.65 \mathrm{GeV} / \mathrm{c}$.

There have been attempts to describe the data 21 23. on inclusive spectra of the open charm production in soft $p p$ collisions and the asymmetry between the $D^{-}$and $D^{+}$production in $\pi^{-} p$ collisions within the string model [24, 25] without the assumption on the intrinsic charm in a nucleon. However, the description of data is very sensitive to the fragmentation functions of $c$-quarks to charmed hadrons, the knowledge of which up to now is not sufficient, see, e.g. Refs. 26 28. Moreover, the experimental data on the open charm production, for example, $\Lambda_{\mathrm{c}}^{+}$in $p p$ collision at the ISR energies and large $x$, have large uncertainty [29, 30].

The existence of the intrinsic heavy quark components can be observed not only in the forward kinematic region, but also in hard $p p$ processes of photon or vector boson production in association with the heavy flavor jets. The first hint on this was observed at the Tevatron $(\sqrt{s}=1.96 \mathrm{TeV})$ in the production of prompt photons accompanied by heavy jets $(c$ or $b)$ 31 35. The basic underlying hard subprocesses are $g c \rightarrow \gamma c$ and $g b \rightarrow \gamma b$. The measurements of $p p \rightarrow b \gamma X$ at high $p_{\mathrm{T}}$ are consistent with standard analyses where the $b$ quark PDF arises from gluon splitting $g \rightarrow b \bar{b}$. However, the $p p \rightarrow c \gamma X$ data show a significant excess, indicating that the charm PDF has significant support at large $x$. This is consistent with the fact that the ratio of intrinsic bottom to 
charm probabilities in the proton scales as $m_{\mathrm{c}}^{2} / m_{\mathrm{b}}^{2}[36$.

It was observed that the ratio between the experimental spectrum of the

of their momentum $x_{\mathrm{F}}>0.5$, therefore, in principle, the intrinsic heavy quark signal in such observables could be visible. However, a high experimental accuracy is needed to observe the enhancement in the heavy hadron spectra due to 
the IC or IB contribution to the proton PDF.

\subsection{Outline of this Review}

The review consists of 7 sections. In Section 2 we present a more detailed overview of the concepts of extrinsic and intrinsic quark components in a nucleon and their distinction. In Section 3 we show why the intrinsic quark components can be visible in the hard $p p$ processes at high energies. Section 4 is devoted to the intrinsic strangeness (IS) content of the nucleon and search for it in hard $p p$ collisions at high energies. Section 5 is a short overview of the global analysis of PDFs with intrinsic charm. Section 6 is devoted to search for the intrinsic heavy quark in processes at collider energies, namely, in the inclusive production of open charm or Higgs boson and production of prompt photons $\gamma$ or gauge vector bosons $W / Z$ accompanied by $c(b)$-jets or open charm in $p p$ collision at LHC energies. There we discuss a possible test of the IC contribution in proton at LHC. Finally, in Section 7 we present the conclusion.

\section{Extrinsic and intrinsic quark components in nucleon}

By definition, the $\operatorname{PDF} f_{\mathrm{a}}(x, \mu)$ is a function of the proton momentum fraction $x$ carried by parton $a$ (quark $q$ or gluon $g$ ) at the QCD momentum transfer scale $\mu$. For small values of $\mu$, corresponding to the long distance scales greater than $1 / \mu_{0}$, the PDF cannot be calculated from the first principles of QCD (although some progress in this direction has been recently achieved within the lattice methods [44, 45]). If the $\operatorname{PDF} f_{\mathrm{a}}(x, \mu)$, is known at a scale $\mu>\mu_{0}$, one can calculate it at any other scale $\mu$ by solving the perturbative QCD evolution equations (DGLAP) 1 1 3] usually, the value of the starting scale $\mu_{0}$ is chosen about a few $\mathrm{GeV} / \mathrm{c}$. As the input of evolution functions $f_{\mathrm{a}}\left(x, \mu_{0}\right)$ can be taken at any scale the functions can be found empirically from some "QCD global analysis" 46 48, of a large variety of data, typically at $\mu>\mu_{0}$.

In general, almost all $p p$ processes that took place at the LHC energies, including the Higgs boson production, are sensitive to the charm $f_{\mathrm{c}}(x, \mu)$ or 
bottom $f_{\mathrm{b}}(x, \mu)$ PDFs. Heavy quark become visible in proton at scales $\mu_{\mathrm{c}}$ and $\mu_{\mathrm{b}}$ and their contents increases with increasing $Q^{2}$-scale through the gluon splitting in the DGLAP $Q^{2}$ evolution [1]. Direct measurement of the open charm and open bottom production in the deep inelastic processes (DIS) confirms the QCD picture of heavy quarks dynamics in proton [12].

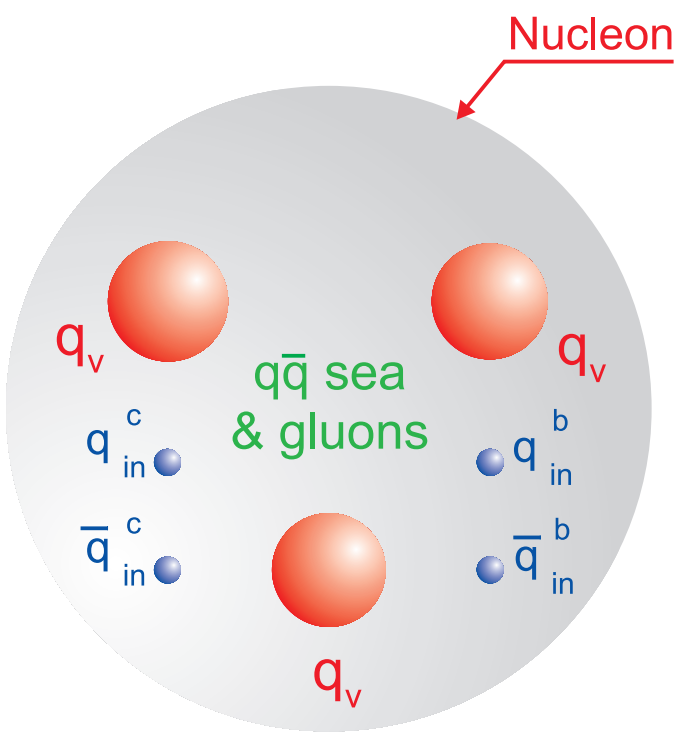

Figure 1: Schematic presentation of a proton during the $p p$ collision during the $p p$ collision at a value of $Q^{2}$, when the lifetime of the intrinsic Fock-state is much larger than the interaction time.

As was assumed in [6, 13, there are extrinsic and intrinsic contributions to the quark-gluon structure of the proton. The extrinsic (or perturbative sea) quarks and gluons are generated on a short time scale associated with a large transverse momentum processes as a result of gluon dynamics. Their distribution functions satisfy the standard QCD evolution equations [1-3. The extrinsic quark contribution $q_{\mathrm{ex}}(x)$ to PDFs is mostly significant at low $x$ and decreases when the quark momentum fraction grows. It depends logarithmically on the heavy quark mass $M_{\mathrm{Q}}$, while the intrinsic quark contribution $q_{\text {in }}(x)$, the residual part of the proton structure not coming from the perturbative gluon splitting, is almost zero at low $x$ and dominates compared to the extrinsic one at large 
$x>0.1$. It depends on the heavy quark mass as $1 / M_{\mathrm{Q}}^{2}$. Initially in [6, 13] authors have proposed existence of the 5-quark state in a proton, which consists of valence $u u d$ quarks and the charm-anticharm pair $c \bar{c}$, i.e., $|u u d c \bar{c}\rangle$. This

flavor intrinsic $Q \bar{Q}$ pairs in a nucleon.

According to Fig. 2, if the gluon-gluon scattering box diagram, $g g \rightarrow Q \bar{Q} \rightarrow$ $g g$, is inserted into the proton self-energy graph, the cut of this amplitude generates five-quark Fock states of the proton $|u u d Q \bar{Q}\rangle[15]$.

225 $b \bar{b}$ within the proton state could be due to the diagrams where heavy quarks are multiply connected to valence quarks. On this basis, within the MIT bag 


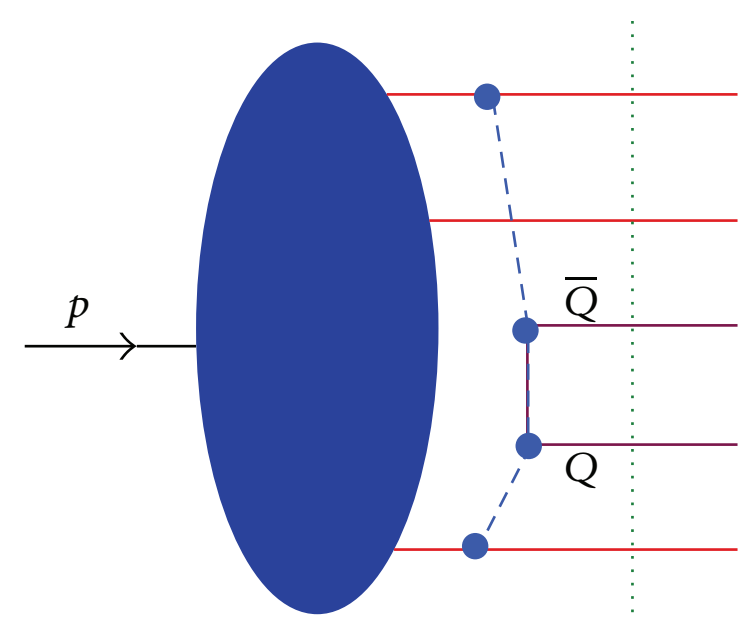

Figure 2: Schematic graph of the $Q \bar{Q}$ pair creation in a nucleon.

model [51], the probability to find the five-quark component $|u u d c \bar{c}\rangle$ bound within the nucleon bag was estimated to be about $1-2 \%$. Later some other models were also developed [16, 50, 52, 55]. One of them considered a quasi-twobody state $\bar{D}^{0}(u \bar{c}) \bar{\Lambda}_{\mathrm{c}}^{+}(u d c)$ in the proton [16]. In [16, 50, 56] the probability to find the intrinsic charm (IC) in the proton (the weight of the relevant Fock state in the proton) was assumed to be $1-3.5 \%$. The probability of the intrinsic bottom (IB) in the proton is suppressed by the factor $m_{\mathrm{c}}^{2} / m_{\mathrm{b}}^{2} \simeq 0.1$ [36, where $m_{\mathrm{c}}$ and $m_{\mathrm{b}}$ are the masses of the charmed and bottom quarks.

The probability distribution for the 5-quark state $(|u u d c \bar{c}\rangle)$ in the light-cone description of the proton was first calculated in [6]. The general form for this distribution calculated within the light-cone dynamics in the so-called BHPS model [6, 13] can be written as [16]

$$
\mathrm{d} P=N \prod_{j=1}^{5} \frac{\mathrm{d} x_{j}}{x_{j}} \delta\left(1-\sum_{j=1}^{5} x_{j}\right) \prod_{j=1}^{5} \mathrm{~d}^{2} p_{\mathrm{T} j} \delta^{(2)}\left(\sum_{j=1}^{5} p_{\mathrm{T} j}\right) \frac{F^{2}(s)}{\left(s-m_{\mathrm{N}}^{2}\right)^{2}},
$$

240 where

$$
s=\sum_{j=1}^{5} \frac{p_{\mathrm{T} j}^{2}+m_{j}^{2}}{x_{j}}
$$

and $x_{j}$ is the longitudinal momentum fraction of the parton, $m_{j}$ is its mass and 
$m_{\mathrm{N}}$ is the nucleon mass. The form factor $F^{2}$ characterizes the dynamics of the bound state. It suppresses the contributions at large values of $p_{\mathrm{T} j}$ and small $x_{j}$ to make the integrated probability $P$ converge.

LFWFs (light front wave functions) are defined at a fixed LF (light front) time $\tau=t+z / c$. They are arbitrarily off-shell in $P^{-}=P^{0}-P^{3}$ and thus invariant mass $W$.

The intrinsic heavy quark LF Fock states such as $|u u d Q \bar{Q}\rangle$ arise from standard QCD - any diagram where the $Q \bar{Q}$ is multi-connected to the valence Fock states.

The simplest IQ Fock states correspond to the insertion of gluon-gluon scattering $g g \rightarrow Q \bar{Q} \rightarrow g g$ in the hadron self-energy. Its cut diagram is presented in Fig. 2,

The LFWFs are maximal at minimal off-shellness; i.e., minimum invariant mass. This occurs when all of the constituents have the same rapidity and thus $x_{i} \propto \sqrt{m_{i}^{2}+k_{\mathrm{T} i}^{2}}$. This is why the heavy partons have the highest LF $x_{i}$.

Even in QED one has intrinsic heavy lepton states such as $\left|e^{+} e^{-} \mu^{+} \mu^{-}\right\rangle$LF Fock states. In the Abelian case the intrinsic heavy lepton probability falls as $\frac{1}{\mathcal{M}^{4}}$, where $\mathcal{M}$ is the effective mass of this system. In contrast, the probability falls as $\frac{1}{\mathcal{M}^{2}}$ in non-Abelian QCD. Thus one has a direct way to validate QCD.

One could actually measure the $x$ of distributions of such Fock states in positronium by colliding and dissociating relativistic positronium atoms in a gas jet target: $\left[e^{+} e^{-}\right] Z \rightarrow e^{+} e^{-} \mu^{+} \mu^{-} Z$.

This is the analog of the Ashery experiment $\pi A \rightarrow q \bar{q} A$ which measured the LFWF of the pion.

Since the quarks in $|u u d Q \bar{A}\rangle$ tend to have the same rapidity, they will continue to reinteract. This causes the $s(x) \bar{s}(x)$ asymmetry in the proton PDF.

IQ is rigorous - it is a first-principle prediction of QCD. It can be analysed using the OPE as shown by M. Polyakov et al. [36] and earlier by paper from S.J. Brodsky et al. [4].

The production cross-section for $\gamma^{*} p \rightarrow c \bar{c}$ vanishes as a power of $W^{2}-W_{\text {threshold }}^{2}$. This is why one cannot reliably use SLAC data to test IC, as noted by Gardner 
and Brodsky. Very few events satisfy this cut [57.

The IQ states will be produced at small rapidity $\Delta y$ relative to the SMOG nuclear target in the LHCb fixed target experiment [58. This provides an amazing testing ground for IQ.

One can thus validate the ISR experiments which measured $\Lambda_{\mathrm{c}}$ and $\Lambda_{\mathrm{b}}$ at high $x_{\mathrm{F}}$ [29, 30]. This leading particle phenomena was also observed by SELEX [59] in $p A \rightarrow \Lambda_{\mathrm{c}} X$.

A dramatic consequence of IQ: Higgs boson production at high $x_{\mathrm{F}}>0.8$ at the ISR. This is supported by the measurement of $J / \psi$ by Badier et al. (NA3) and even double $J / \psi$ hadroproduction at high $x_{\mathrm{F}}$ [41, 42, 60].

Neglecting all the quark masses in comparison with the nucleon mass and the charm quark mass $m_{\mathrm{c}}$, and integrating (1) over $\mathrm{d} x_{1} \ldots \mathrm{d} x_{4}$ and putting $F^{2}=1$ 285 in Eq. (1) one can get the following probability to find the intrinsic charm with momentum fraction $x \equiv x_{5}$ in the nucleon 61 .

$$
P(x)=\frac{N x^{2}}{6(1-c x)^{5}}\left(\phi_{1}(x)+\phi_{2}(x)[\ln (x)-\ln [1-c(1-x) x]]\right),
$$

where $x=x_{5}, c=m_{\mathrm{N}}^{2} / m_{\mathrm{c}}^{2}$,

$$
\phi_{1}(x)=(1-x)(1-c x)[1+x[10+x-c(1-x)(x(10-c(1-x))+2)]],
$$

and

$$
\phi_{2}(x)=6 x[1+x(1-c(1-x))][1-c(1-x) x] .
$$

Here $N$ is found from the normalization equation:

$$
\int_{0}^{1} P(x) \mathrm{d} x=w,
$$
the BHPS result [6, 16, 61]:

$$
P(x)=600 w x^{2}\left[6 x(1+x) \ln x+(1-x)\left(1+10 x+x^{2}\right)\right],
$$

Equation (7) was first derived by Brodsky, Hoyer, Peterson and Sakai [6] and, usually, this approach is called as the BHPS model. In principle, the form factor 
$F^{2}$ can be less than 1. It is due to the suppression of the high mass configurations 16:

$$
F^{2}=\exp \left(\frac{-\left(s-m_{\mathrm{N}}^{2}\right)}{\Lambda^{2}}\right)
$$

or the power-low suppression factor:

$$
F^{2}=\frac{1}{\left(s+\Lambda^{2}\right)^{n}}
$$

As is shown in [16, the results for $P(x)$ using the exponential or power-low suppression factor with $n=4$ are rather similar to the BHPS model, but are somewhat smaller at $x>0.5$. Values $n \leq 2$ are unphysical because they result in the total probability divergence [16 and the use Eq. (9) at $n=3$ leads to a dependence $F^{2} \sim 1 / m_{\mathrm{c}}^{2}$ that is similar to the result of [5].

Another way to suppress the high-mass Fock space components can be made on the basis of quasi-two-body states [16, 49]. In particular, the relevant 5-quark Fock configurations can be grouped as $(u d c)(u \bar{c})$ considered as the off-shell twobody state $\bar{D}^{0} \Lambda_{\mathrm{c}}^{+}$. In this case the factor $F^{2}$ is chosen, for example, in [16] as power-low suppression and can be different for $\bar{D}^{0}$ and $\Lambda_{\mathrm{c}}^{+}$. It leads to the asymmetry between the $c$ and $\bar{c}$-distribution, which was considered first in [49. The observation of a $c(x)-\bar{c}(x)$ difference can definitively prove a non-perturbative charm quark component since the $c \bar{c}$ pairs produced by gluon splitting are symmetric, according to the NLO calculations, whereas the NNLO calculations can give different shapes for $c(x)$ and $\bar{c}(x)$, see for example, [16] and 62. The charm and anti-charm distributions obtained within the cloudmodel and can be parametrized by the following form at $\mu^{2}=m_{\mathrm{c}}^{2}[50$ :

$$
c(x)=N x^{1.897}(1-x)^{6.095} ; \quad \bar{c}(x)=\bar{N} x^{2.511}(1-x)^{4.929},
$$

315

where $N / \bar{N}$ is determined by the quark number sum rule [50]:

$$
\int_{0}^{1}[c(x)-\bar{c}(x)] \mathrm{d} x=0,
$$


and the normalization factor is related to the IC probability. In fact, the asymmetry between $\bar{\Lambda}_{\mathrm{c}}^{-}$and $\Lambda_{\mathrm{c}}^{+}$baryons was observed by the SELEX Collaboration 63] in $\Sigma^{-} p$ collision at the beam momentum about $600 \mathrm{GeV} / \mathrm{c}$. This asymmetry was about $50 \%$ at $x_{\mathrm{F}} \simeq 0.3$. However, in $\pi^{-} p$ and $p p$ collisions the asymmetry was practically invisible because of the very poor statistics.

For the strange-antistrange pairs asymmetry similar to the charm-anticharm one was predicted in [49] and [54] within the light-cone five meson-baryon fluctuation model assuming hypothesis about the intrinsic strangeness in a nucleon. The difference between $c(x)$ and $\bar{c}(x)$ was studied, for example, in [49. Later we will discuss a possible verification of this quark-antiquark asymmetry of the nucleon sea at the LHC.

Alternatively, the relevant 5-quark configurations can be grouped as $(u u d)(c \bar{c})$ or the off-shell proton and $J / \Psi$-meson [16]. However, this quark combination does not result in the $c-\bar{c}$ asymmetry.

In contrast to the light-cone approach of heavy flavor Fock quark components in a nucleon, the alternative purely phenomenological scenario was suggested [50]. In this model the charm distribution in a nucleon is sea like, i.e., similar to that of the light-flavor sea quarks $c(x)=\bar{c}(x)=\bar{d}(x)+\bar{u}(x)$ at $\mu_{0}^{2}=m_{\mathrm{c}}^{2}$.

335 The initial non-perturbative forms for $c(x)$ and $\bar{c}(x)$ at $\mu_{0}$ specified above are usually used as inputs to the general-mass perturbative DGLAP evolution. The global analysis of distributions of the extrinsic and intrinsic heavy-flavor and light-flavor quark distributions and gluons was performed in [50] on basis of the BHPS, the meson-cloud model, sea like scenario and the PDF of type redraw the figures from [50 and [16, but let us discuss the main properties of these parton distributions as a function of $x$ at different values of scale $\mu$. The BHPS model for the $x$ distribution for intrinsic heavy flavor quarks was derived within the light-cone approach. The derivation uses some additional simplifying approximations, which can be modified. The shape of the intrinsic quark distribution versus $x$ is due to its dependence on the energy propagator 
$1 /\left(s-m_{\mathrm{N}}^{2}\right)$ in Eq. 11). Using the DGLAP $\mu^{2}$-evolution for parton distributions it was shown that the intrinsic charm provides the dominant contribution to $c(x)$ and $\bar{c}(x)$ at any $\mu$, if the form of the IC distribution is given by the BHPS model with the non-zero IC probability $w$. The comparison of the charm distribution to other flavor distributions presented, for example, in [50] showed that the inclusion of the IC contribution with $w=1$ and $3.5 \%$ leads to an enhancement, which is larger than the values of light sea quark distributions $\left(u_{\text {sea }}=\bar{u}_{\text {sea }}\right.$, $\left.d_{\text {sea }}=\bar{d}_{\text {sea }}, s_{\text {sea }}=\bar{s}_{\text {sea }}\right)$ at $x>0.4$ at any QCD scale $\mu$. The intrinsic charm contribution $c_{\mathrm{in}}(x)$ dominates compared to the extrinsic one $c_{\mathrm{ex}}(x)$ at $x>0.1$. The shape of the charm quark distribution $c(x)=c_{\mathrm{ex}}(x)+c_{\mathrm{in}}(x)$ is similar to the valence quark distribution, but is smaller by a factor of about 10 in the whole region of $0.003<x<1$ at low $\mu$ and in the region of $x>0.1$ at large $\mu$. The results for the IC distribution using the BHPS model and the $D_{0} \Lambda_{\mathrm{c}}^{+}$mesoncloud model are close [50. The sea like scenario results in no any enhancement for the charm quark distribution at $x>0.1$, as it is within the BHPS and the meson-cloud models. However, the sea like model gives for $c(x)$ an excess about a factor of $1.5-2$ compared to the $c_{\mathrm{ex}}(x)$ at $0.003<x<0.1$ and low $\mu$ and there is no excess at large $\mu \geq 100 \mathrm{GeV} / \mathrm{c}$.

\subsection{Intrinsic charm density in a proton as a function of IC probability $w$}

According to [15, 64, 65, the intrinsic charm distribution at the starting scale $\mu_{0}^{2}$ as a function of $x$ can be presented in the following approximated form similar to Eq. 7

$$
c_{\text {int }}\left(x, \mu_{0}^{2}\right)=c_{0} w x^{2}\left[(1-x)\left(1+10 x+x^{2}\right)+6 x(1+x) \ln (x)\right],
$$

where $w$ is the probability to find the Fock state $|u u d c \bar{c}\rangle$ in the proton, $c_{0}$ is the normalization constant and the masses of the light quarks and the nucleon are negligible compared to the charm quark mass. The inclusion of the non-zero nucleon mass leads to a more complicated analytic form [50, 61]. According to the BHPS model [6, 13, the charm density in a proton is the sum of the 
extrinsic and intrinsic charm densities,

$$
x c\left(x, \mu_{0}^{2}\right)=x c_{\mathrm{ext}}\left(x, \mu_{0}^{2}\right)+x c_{\mathrm{int}}\left(x, \mu_{0}^{2}\right) .
$$

constant in front of the function dependent on $x$ and $\mu^{2}$, one can suggest a 
simple relation at any $w \leq w_{\max }$ :

$$
x c_{\mathrm{int}}\left(x, \mu^{2}\right)=\left.\frac{w}{w_{\max }} x c_{\mathrm{int}}\left(x, \mu^{2}\right)\right|_{w=w_{\max }} .
$$

Actually, Eq. 14 is the linear interpolation between two charm densities at the scale $\mu^{2}$, obtained at $w=w_{\max }$ and $w=0$. Later we adopt the charm dis-

tribution function from the CTEQ66M set [56]. We assume $w_{\max }=3.5 \%$ everywhere, which corresponds to the CTEQ66c1 set [56].

The charmed quark densities at different $w$ and $\mu^{2}$ are shown in Fig. 3.

Our results are in good agreement with the calculations 64 but are obtained in the more straightforward manner. Additionally, we performed the three-point interpolation of the charmed quark distributions (over $w=0, w=1 \%$ and $w=3.5 \%$, which correspond to the CTEQ66M, CTEQ66c0 and CTEQ66c1 sets, respectively). These results differ from the ones based on 14 by no more than $0.5 \%$, thus giving us the confidence in our starting point. The comparison to CT14 with IC $1 \%$ and $2 \%$ was also performed and it is in very good agreement with results obtained using Eq. (14). We conclude that the sensitivity of resulting distributions to choice of intrinsic charm PDF (CTEQ66 or CT14) is very small.

Below we apply the charmed quark density obtained by (13) and (14) to calculate the total and differential cross-sections of associated prompt photon $\gamma$ or $Z$ boson and heavy flavor jet production, $\gamma(Z)+Q$, at the LHC conditions. The suggested procedure to calculate $x c_{\text {int }}\left(x, \mu^{2}\right)$ at any $w \leq w_{\max }$ allows us to reduce significantly the time for the calculation of these observables.

As a rule, the gluons and sea quarks play the key role in hard processes of open charm hadroproduction. Simultaneously, due to the non-perturbative intrinsic heavy quark components one can expect some excess of these heavy quark PDFs over the ordinary sea quark PDFs at $x>0.1$. Therefore the existence of this intrinsic charm component can lead to some enhancement in the inclusive spectra of open charm hadrons, in particular $D$-mesons, produced at the LHC in $p p$-collisions at large pseudo-rapidities $\eta$ and large transverse momenta $p_{\mathrm{T}}$ [66]. Furthermore, as we know from [6, 13, 16, 150, 51, 56] photons 

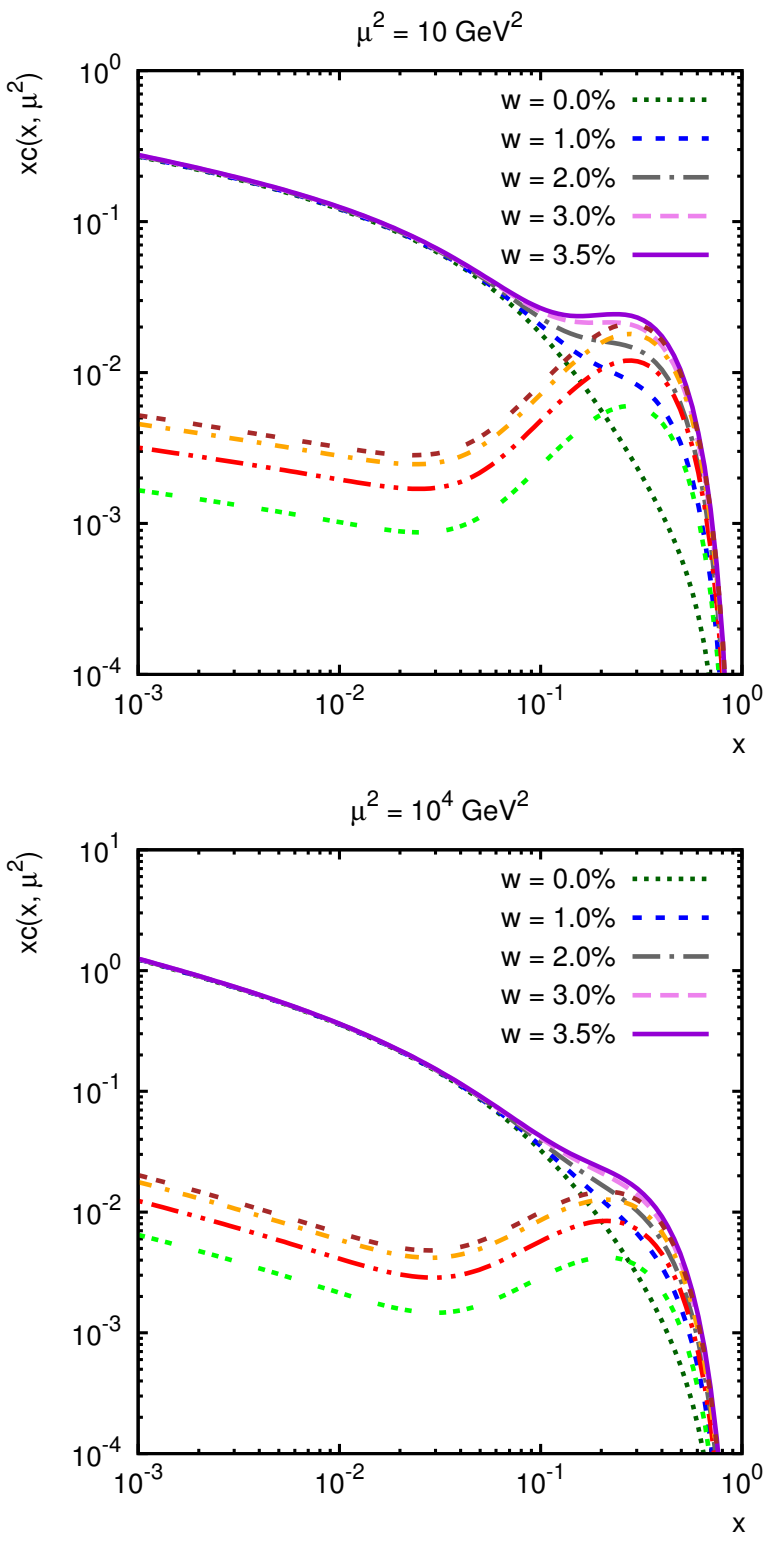

Figure 3: The total charmed quark density $x c\left(x, \mu^{2}\right)$ as a function of $x$ at different values of $w$ at $\mu^{2}=10(\mathrm{GeV} / \mathrm{c})^{2}$ (top) and $\mu^{2}=10^{4}(\mathrm{GeV} / \mathrm{c})^{2}$ (bottom). The triple-dashed line is the IC contribution at $w=1 \%$, the dashed-double-dotted line corresponds to the IC at $w=2 \%$, the dashed-dotted curve is the IC at $w=3 \%$ and the double-dashed line corresponds to the IC at $w=3.5 \%$. 
produced in association with heavy quarks $Q(\equiv c, b)$ in the final state of $p p$ collisions provide valuable information about the parton distributions in the proton [16, 31, 34, 36, 38, 50, 53, 56, 66, 74.

In this paper, having in mind these considerations we will first discuss where the above-mentioned heavy flavor Fock states in the proton could be searched for at the LHC energies. Following this we analyze in detail, and give predictions for, the LHC semi-inclusive $p p$-production of prompt photons accompanied by $c$-jets including the intrinsic charm component in the PDF.

\section{Intrinsic heavy quarks and hard $p p$ collisions}

\subsection{Where can one look for the intrinsic heavy quarks?}

It is known that in the open charm/bottom $p p$-production at large momentum transfer the hard QCD interactions of two sea quarks, two gluons and a gluon with a sea quark play the main role. The cross section for hard inclusive hadronic reactions $p p \rightarrow h X$ can be factorized [75] as the product of structure and fragmentation functions convoluted with the sum of contributing $2 \rightarrow 2$ quark and gluon subprocess $i+j \rightarrow i^{\prime}+j^{\prime}$ cross sections. This result can be presented in the following general form [76] (see also [77):

$$
E \frac{\mathrm{d} \sigma}{\mathrm{d}^{3} p}=\sum_{i, j} \int \mathrm{d}^{2} k_{\mathrm{T} i} \int \mathrm{d}^{2} k_{\mathrm{T} j} \int_{x_{i}^{\min }}^{1} \mathrm{~d} x_{i} \int_{x_{j}^{\min }}^{1} \mathrm{~d} x_{j} f_{i}\left(x_{i}, k_{\mathrm{T} i}\right) f_{j}\left(x_{j}, k_{\mathrm{T} j}\right) \frac{\mathrm{d} \sigma_{i j}(\hat{s}, \hat{t})}{\mathrm{d} \hat{t}} \frac{D_{i, j}^{\mathrm{h}}\left(z_{\mathrm{h}}\right)}{\pi z_{\mathrm{h}}} .
$$

Here $k_{i, j}$ and $k_{i, j}^{\prime}$ are the four-momenta of the partons $i$ or $j$ before and after the elastic parton-parton scattering, respectively; $k_{\mathrm{T} i}, k_{\mathrm{T} j}$ are the transverse momenta of the partons $i$ and $j ; z$ is the fraction of the hadron momentum from the parton momentum; $f_{i, j}$ is the PDF; and $D_{i, j}$ is the fragmentation function (FF) of the parton $i$ or $j$ into a hadron $h$.

When the transverse momenta of the partons are neglected in comparison with the longitudinal momenta, the variables $\hat{s}, \hat{t}, \hat{u}$ and $z_{\mathrm{h}}$ can be presented in the following forms [75]:

$$
\hat{s}=x_{i} x_{j} s, \quad \hat{t}=x_{i} \frac{t}{z_{\mathrm{h}}}, \quad \hat{u}=x_{j} \frac{u}{z_{\mathrm{h}}}, \quad z_{\mathrm{h}}=\frac{x_{1}}{x_{i}}+\frac{x_{2}}{x_{j}},
$$


where

$$
x_{1}=-\frac{u}{s}=\frac{x_{\mathrm{T}}}{2} \cot \frac{\theta}{2}, \quad x_{2}=-\frac{t}{s}=\frac{x_{\mathrm{T}}}{2} \tan \frac{\theta}{2}, \quad x_{\mathrm{T}}=2 \frac{\sqrt{t u}}{s}=2 \frac{p_{\mathrm{T}}}{\sqrt{s}} .
$$

Here as usual, $s=\left(p_{1}+p_{2}\right)^{2}, t=\left(p_{1}-p_{1}^{\prime}\right)^{2}, u=\left(p_{2}-p_{1}^{\prime}\right)^{2}$, and $p_{1}, p_{2}, p_{1}^{\prime}$ are the 4-momenta of the colliding protons and the produced hadron $h$, respectively; $\theta$ is the scattering angle for the hadron $h$ in the $p p$ c.m.s. The lower limits of the integration in 15 are

$$
x_{i}^{\min }=\frac{x_{\mathrm{T}} \cot \frac{\theta}{2}}{2-x_{\mathrm{T}} \tan \frac{\theta}{2}}, \quad x_{j}^{\min }=\frac{x_{i} x_{\mathrm{T}} \tan \frac{\theta}{2}}{2 x_{i}-x_{\mathrm{T}} \cot \frac{\theta}{2}} .
$$

Actually, the parton distribution functions $f_{i}\left(x_{i}, k_{i T}\right)$ also depend on the fourmomentum transfer squared $Q^{2}$ that is related to the Mandelstam variables $\hat{s}$, $\hat{t}, \hat{u}$ for the elastic parton-parton scattering [75, 76]

$$
Q^{2}=\frac{2 \hat{s} \hat{t} \hat{u}}{\hat{s}^{2}+\hat{t}^{2}+\hat{u}^{2}}
$$

One can see that the Feynman variable $x_{\mathrm{F}}$ of the produced hadron, can be expressed via the variables $p_{\mathrm{T}}$ and $\eta$, or $\theta$ the hadron scattering angle in the $p p$ c.m.s,

$$
x_{\mathrm{F}} \equiv \frac{2 p_{\mathrm{z}}}{\sqrt{s}}=\frac{2 p_{\mathrm{T}}}{\sqrt{s}} \frac{1}{\tan \theta}=\frac{2 p_{\mathrm{T}}}{\sqrt{s}} \sinh \eta .
$$

At small scattering angles of the produced hadron this formula becomes

$$
x_{\mathrm{F}} \sim \frac{2 p_{\mathrm{T}}}{\sqrt{s}} \frac{1}{\theta} .
$$

It is clear that for fixed $p_{\mathrm{T}}$ an outgoing hadron must possess a very small $\theta$ or very large $\eta$ in order to have large $x_{\mathrm{F}}$ (to follow forward, or backward direction).

In the fragmentation region (of large $x_{\mathrm{F}}$ ) the Feynman variable $x_{\mathrm{F}}$ of the produced hadron is related to the variable $x$ of the intrinsic charm quark in the proton, and according to the longitudinal momentum conservation law, the $x_{\mathrm{F}} \simeq x$ (and $\left.x_{\mathrm{F}}<x\right)$. Therefore, the visible excess of the inclusive spectrum, for example, of $K$-mesons can be due to the enhancement of the IS distribution ${ }_{475} \quad$ (see Fig. (4) at $x>0.1$. 
The lower limits of the integration in (15) can be presented in the following form:

$$
x_{i}^{\min }=\frac{x_{\mathrm{R}}+x_{\mathrm{F}}}{2-\left(x_{\mathrm{R}}-x_{\mathrm{F}}\right)}, \quad x_{j}^{\min }=\frac{x_{i}\left(x_{\mathrm{R}}-x_{\mathrm{F}}\right)}{2 x_{i}-\left(x_{\mathrm{R}}+x_{\mathrm{F}}\right)},
$$

where $x_{\mathrm{R}}=2 p / \sqrt{s}$. One can see from $(22)$ that, at least, one of the low limits $x_{i}^{\text {min }}$ of the integral 15 must be $\geq x_{\mathrm{F}}$. Thus if $x_{\mathrm{F}} \geq 0.1$, then $x_{i}^{\min }>0.1$, where

480

the ordinary (extrinsic) charm distribution is completely negligible in comparison with the intrinsic charm distribution. Therefore, at $x_{\mathrm{F}} \geq 0.1$, or equivalently at the charm momentum fraction $x_{\mathrm{c}}>0.1$ the intrinsic charm distribution intensifies the charm PDF contribution into charm hadroproduction substantially (see Fig. 3). As a result, the spectrum of the open charm hadroproduction can be increased in a certain region of $p_{\mathrm{T}}$ and $\eta$ (which corresponds to $x_{\mathrm{F}} \geq 0.1$ in accordance to 22). We stress that this excess (or even the very possibility to observe relevant events in this region) is due to the non-zero contribution of IC component at $x_{\mathrm{c}}>x_{\mathrm{F}}>0.1$ (where non-IC component completely vanishes).

This possibility was demonstrated for the $D$-meson production at the LHC in [66. It was shown that the $p_{\mathrm{T}}$ spectrum of $D$-mesons is enhanced at pseudorapidities of $3<\eta<5.5$ and $10 \mathrm{GeV} / \mathrm{c}<p_{\mathrm{T}}<25 \mathrm{GeV} / \mathrm{c}$ due to the IC contribution, which was included using the CTEQ66c PDF [56]. For example, due to the IC PDF, with probability about $3.5 \%$, the $p_{\mathrm{T}}$-spectrum increases by a factor of 2 at $\eta=4.5$. A similar effect was predicted in [78].

One expects a similar enhancement in the experimental spectra of the open bottom production due to the (hidden) intrinsic bottom (IB) in the proton, which could have a distribution very similar to the one given in (12). However, the probability $w_{\text {IB }}$ to find the Fock state with the IB contribution $|u u d b \bar{b}\rangle$ in the proton is about 10 times smaller than the IC probability $w_{\text {IC }}$ due to relation $w_{\mathrm{IB}} / w_{\mathrm{IC}} \sim m_{\mathrm{c}}^{2} / m_{\mathrm{b}}^{2}[13,36$.

The IC "signal" can be studied not only in the inclusive open (forward) charm hadroproduction at the LHC, but also in some other processes, such as production of real prompt photons $\gamma$ or virtual ones $\gamma^{*}$, or $Z^{0}$-bosons (decaying into dileptons) accompanied by $c$-jets in the kinematics available to the ATLAS 

in the proton.

\section{Intrinsic strangeness in proton}

The BHPS model can be applied also for the search for the intrinsic strangeness (IS), see, for example, 68, where the early HERMES data 69] on the strange quark distribution $x S\left(x, Q_{0}^{2}\right)=x\left[s\left(x, Q_{0}^{2}\right)+\bar{s}\left(x, Q_{0}^{2}\right)\right]$ at $x>0.1$ and $Q_{0}^{2}=2.5(\mathrm{GeV} / \mathrm{c})^{2}$ has described rather satisfactorily using the IS contribution in a form about $2.5 \%$. Unfortunately later the new HERMES data appeared [79. The HERMES information on $x S\left(x, Q_{0}^{2}\right)$ were extracted from the data on the multiplicities of charged $K$-mesons produced in the deep inelastic ep scattering. The extraction of the polarization-averaged strange quark distribution from the HERMES data had an uncertainty related to the fragmentation functions (FFs). In Fig. 4 the old HERMES data 69. (circles) and the new ones (squares and triangles) are presented. The square points in Fig. 4 correspond to the FFs taken from [80] (DSS) and the triangles correspond to the following assumption:

$$
\int D_{\mathrm{S}}^{\mathrm{K}}\left(z, Q_{0}^{2}\right) \mathrm{d} z=1.27,
$$

525 mesons).

The dashed-dotted line in Fig. 4 corresponds to the PDF of type CTEQ6L [46], the dash curve is the IS contribution with its probability $w_{\mathrm{IS}}=2.5 \%$ calculated within the BHPS model [6, 13. One can see from Fig. 4 a large uncertainty of the HERMES data. Therefore, unfortunately the HERMES data do not allow to extract a reliable information on the IS contribution to the PDF of strange quarks. 


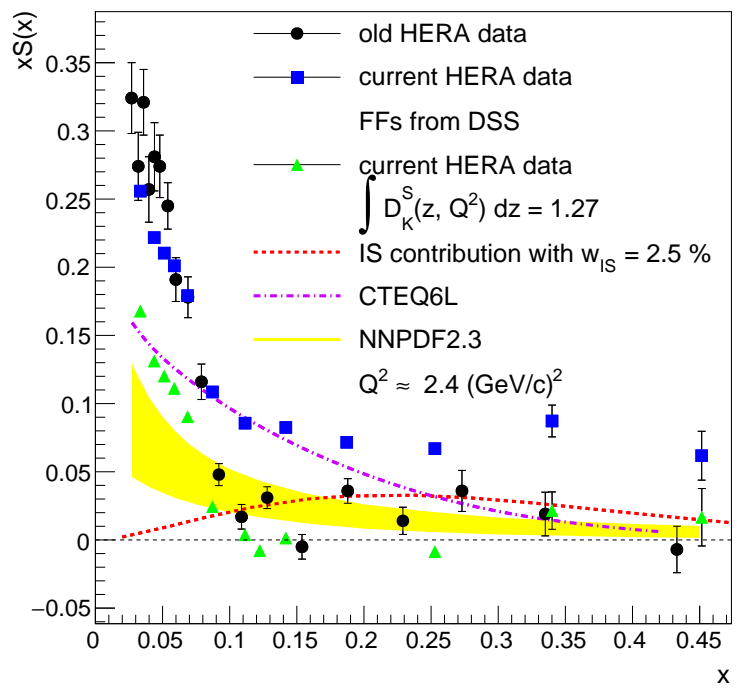

Figure 4: The distributions of strange quarks $x S(x)=x(s(x)+\bar{s}(x))$ in the proton at $Q^{2}=2.4(\mathrm{GeV} / \mathrm{c})^{2}$, the black points are the old HERMES data 69]; the blue squares are the new data 79, when the FFs are taken from [80] (DSS); the green triangles are the new data assuming he normalization given by Eq. 23 ; the dashed curve is the contribution of the intrinsic strangeness (IS) in the proton with the probability $2.5 \%$; the yellow band corresponds to NNPDF2.3 set [19].

Let us analyze now how the possible existence of the intrinsic strangeness in the proton can be visible in $p p$ collisions. For example, consider the $K^{-}$-meson production in the process $p p \rightarrow K^{-}+X$. Considering the intrinsic strangeness in the proton [81] we calculated the inclusive spectrum $\mathrm{d} \sigma / \mathrm{d}^{3} p$ of such mesons within the hard scattering model (Eq. (15)), which describes satisfactorily the HERA and HERMES data on the DIS. The FF and the parton cross-sections were taken from [82, 83, respectively, as mentioned above.

We also emphasize that the intrinsic $s(x)$ and $\bar{s}(x)$ are expected to be different in the proton since the comoving valence and strange quarks in the $|u u d s \bar{s}\rangle$ Fock state can repeatedly interact. This can also be understood by the duality of this Fock state with meson-nucleon fluctuations such as the $K^{+}(\bar{s} u) \Lambda(u d s)$ state. This duality [49] also predicts very different $s(x)$ and $\bar{s}(x)$ spin distribu- 
tions in a polarized proton.

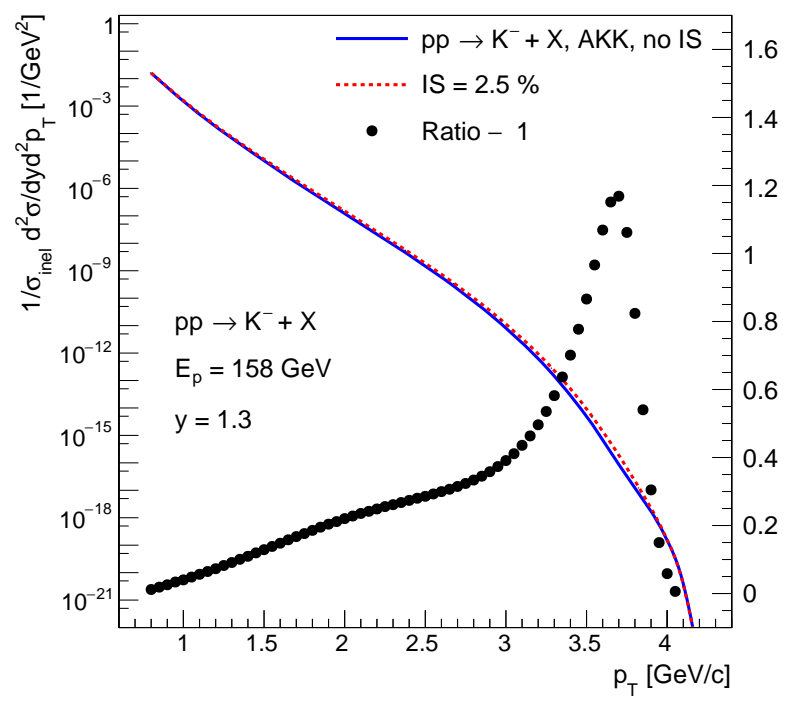

Figure 5: The $\mathrm{K}^{-}$-meson distributions (with and without intrinsic strangeness contribution) over the transverse momentum $p_{\mathrm{T}}$ for $p p \rightarrow K^{-}+X$ at the initial energy $E=158 \mathrm{GeV} / \mathrm{c}$, the rapidity $y=1.3$ and $p_{\mathrm{T}} \geq 0.8 \mathrm{GeV} / \mathrm{c}$.

In Figs. 5 , 6 the inclusive $p_{\mathrm{T}}$-spectra of $K^{-}$-mesons produced in $p p$ collision at the initial energy $E_{p}=158 \mathrm{GeV} / \mathrm{c}$ are presented at the rapidity $|y|=1.3$ (Fig. 5) and $|y|=1.7$ (Fig. 6). The solid lines in Figs. 5 . 6 correspond to our calculation ignoring the intrinsic strangeness (IS) in the proton and the dashed curves correspond to the calculation including the IS with the probability about $2.5 \%$, according to [69]. The dots show the ratio of our calculation with the IS and without the IS minus 1. One can see from Figs. 5. 6 (right axis) that the IS signal can be above $200 \%$ at $|y|=1.3, p_{\mathrm{T}}=3.6-3.7 \mathrm{GeV} / \mathrm{c}$ and slightly smaller, than $200 \%$ at $|y|=1.7, p_{\mathrm{T}} \simeq 2.5 \mathrm{GeV} /$ c. Actually, this is our prediction for the NA61 experiment that is now under way at CERN. 


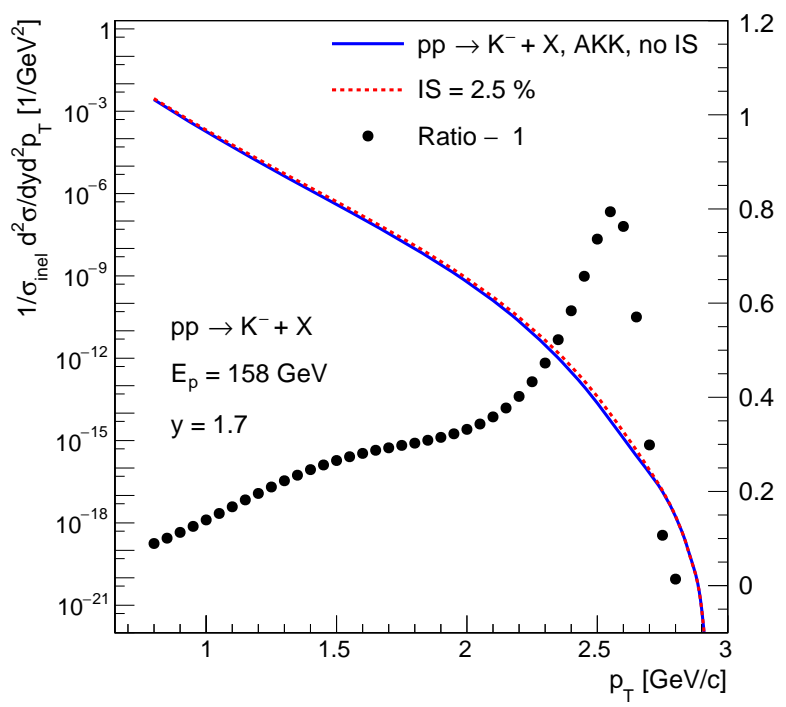

Figure 6: The $K^{-}$-meson distributions (with and without intrinsic strangeness contribution) over the transverse momentum $p_{\mathrm{T}}$ for $p p \rightarrow K^{-}+X$ at the initial energy $E=158 \mathrm{GeV} / \mathrm{c}$, the rapidity $|y|=1.7$ and $p_{\mathrm{T}} \geq 0.8 \mathrm{GeV} / \mathrm{c}$.

\section{Global analysis of PDFs with intrinsic charm}

The charm content of the proton can be studied by the global analysis of PDFs, the charm quark distributions are generated perturbatively by pair radiation off gluons and light quarks, vanishing at a scale about the charm mass $m_{\mathrm{c}}$, see details in [15, 18, 19, 84]. In contrast to this, it was found in [18, that the fitted charm PDF vanishes within uncertainties at a scale $Q \sim 1.5 \mathrm{GeV} / \mathrm{c}$ at $x \leq 0.1$ independently of the $m_{\mathrm{c}}$ value. However, It was also shown [18] that, at $x \geq 0.1$ and low scales the charm PDF does not vanish and rather has an intrinsic component, very weakly scale dependent and almost independent of the $m_{\mathrm{c}}$ value, carrying about $1 \%$ of the total proton momentum. The uncertainties in all other PDFs are slightly increased by including the IC charm, while the dependence of these PDFs on $m_{\mathrm{c}}$ is significantly reduced [18. As was shown, the uncertainties in the fitted charm PDF are reduced if the EMC charm structure function data set is included. The main application of the results obtained 
$m_{\mathrm{c}}$ persists at high scales.

The charm PDF can have a non-vanishing intrinsic component of the nonperturbative origin, see, for example, [15]. On the other hand, if one assumes that the charm PDF is purely perturbative in origin, it vanishes below its proto the charm pole mass, which is not known very precisely. Even if the charm is entirely perturbative and its production threshold is known, there is a problem of getting the accurate predictions because the cross-section of the massive charm is known within the low perturbative order. These difficulties are solved, for example, within the NNPDF3.0 set [19]. Within this set the charm PDF is parametrized on the basis of light quark and gluon PDFs, i.e., with an independent neural network with 37 free parameters.

\section{Intrinsic heavy quark signal in processes at collider energies}

\subsection{Inclusive production of charmed meson}

It was shown that the IC could result in a sizable contribution to the forward charmed meson production [67]. Furthermore the IC "signal" can constitute almost $100 \%$ of the inclusive spectrum of $D$-mesons produced at high pseudo-rapidities $\eta$ and large transverse momenta $p_{\mathrm{T}}$ in $p p$ collisions at LHC energies [66].

590

If the distributions of the intrinsic charm or bottom in the proton are hard enough and are similar in the shape to the valence quark distributions (have the valence-like form), then the production of the charmed (bottom) mesons or charmed (bottom) baryons in the fragmentation region should be similar to the production of pions or nucleons. However, the yield of this production depends on the probability to find the intrinsic charm or bottom in the proton, but this yield appears to be rather small. The PDF which included the IC contribution in the proton have already been used in the perturbative QCD calculations in $[16,50,56$. 
The possible existence of the intrinsic charm in the proton can lead to some enhancement in the inclusive spectra of the open charm hadrons, in particular $D$-mesons, produced at the LHC in $p p$-collisions at high pseudo-rapidities $\eta$ and large transverse momenta $p_{\mathrm{T}}[66$.

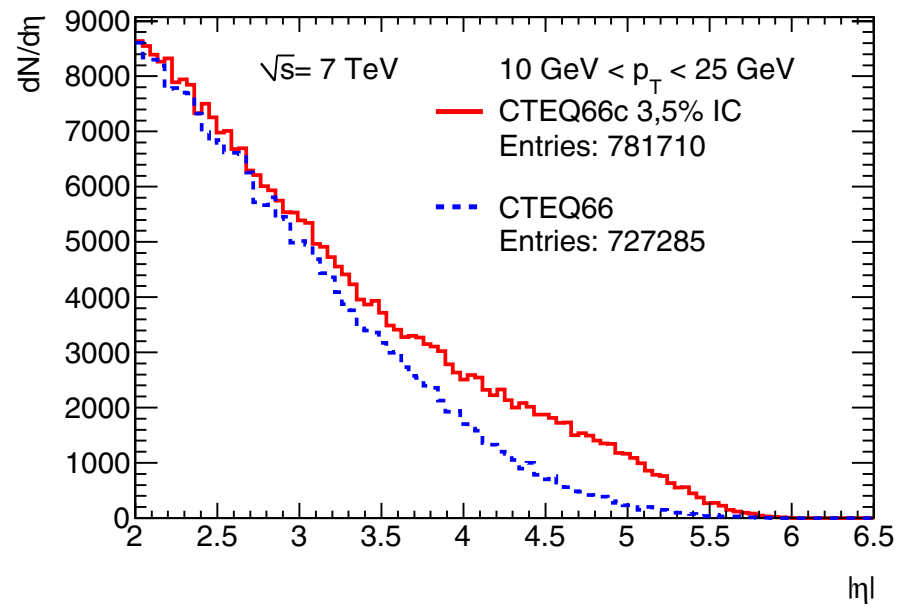

Figure 7: The $D_{0}+\bar{D}_{0}$ distributions over the pseudorapidity $\eta$ in $p p \rightarrow\left(D_{0}+\bar{D}_{0}\right) X$ at $\sqrt{s}=7 \mathrm{TeV} / \mathrm{c}$ and $10 \leq p_{\mathrm{T}} \leq 25 \mathrm{GeV} / \mathrm{c}[66]$.

The inclusion of the intrinsic bottom or/and charm in the proton can increase the yield of the relevant heavy flavour baryons by a factor of 3 to 10 . In particular, we considered a possibility of measuring the reaction $p p \rightarrow \Lambda_{\mathrm{c}}^{+} X \rightarrow$ $\Lambda^{0} \pi^{+} X \rightarrow n \pi^{0} \pi^{+} X$ using the ATLAS, more specifically, one of its forward detectors the ZDC. This measurement can provide information on the intrinsic charm in the proton, the probability of which is estimated to be a factor of 10 higher than the one for the intrinsic bottom in the proton. Finally, it is worth noticing that any reliable non-observation of this enhancement in the experiments at the LHC can severely constrain the intrinsic heavy quark hypothesis.

Our calculations of the charmed meson production in $p p$ collisions were done within the MC generator PYTHIA8 including the IC contribution with the probability about $3.5 \%$ to the PDF are presented in Fig. 766. It is the distribution of the single $D$-mesons $\left(D_{0}+\bar{D}_{0}\right)$ produced in the $p p$ collision at $\sqrt{s}=7 \mathrm{TeV} / \mathrm{c}$ 
as a function of their pseudo-rapidity $\eta$. We found that the contribution of the intrinsic charm in the proton could be studied in the production of $D$-mesons in $p p$ collisions at the LHC. The IC contribution for the single $D^{0}$-meson production can be sizable, it is about $100 \%$ at large rapidities $3 \leq|y| \leq 4.5$ and large transverse momenta $10 \leq p_{\mathrm{T}} \leq 25 \mathrm{GeV} / \mathrm{c}$. As it is shown in [66], for the double $D_{0}$ production this contribution is not larger than $30 \%$ at $p_{\mathrm{T}} \geq 5 \mathrm{GeV} / \mathrm{c}$ and $3 \leq|y| \leq 4.5$. These IC contributions for the single and double $D$-meson production were obtained with the probability of the intrinsic charm taking to be $w_{\mathrm{c} \overline{\mathrm{c}}}=3.5 \%$ [56], and they will decrease by a factor of 3 when $w_{\mathrm{c} \overline{\mathrm{c}}} \simeq 1 \%$. Therefore, this value can be verified experimentally at LHCb.

The predictions presented in Fig. 7 66 could be verified at the LHCb experiment in the kinematic region mentioned above to observe a possible signal for the intrinsic charm. The intrinsic bottom in the proton is suppressed by a factor of 10 [36], therefore its signal in the inclusive spectra of $B$-mesons will probably be very weak.

The IC contributions could be also observed at the SMOG experiment 85] of the $\mathrm{LHCb}$ in the production of the open charm, for example, $D$-mesons or charmed baryons $\Lambda_{\mathrm{c}}$. One can use a gas jet target in a LHC detector - such as the SMOG target available at LHCb - to study novel intrinsic heavy quark 635 physics phenomena in $p A$ collisions. Remarkably, heavy-quark hadrons such as the $\Lambda_{\mathrm{b}}$, double-charm baryons, and exotic hadrons such as tetraquarks and pentaquarks containing heavy quarks will be produced at small target rapidities - nearly at rest in the nuclear target rest frame - and thus can be easily observed. We note that the intrinsic heavy quark Fock states of a proton in the nuclear target, such as $|u u d Q \bar{Q}\rangle$, have high light-front momentum fraction $x_{\mathrm{Q}}$. The collision materializes the far off-shell light-front wave functions of Fock states, such as $|u u d b \bar{b}\rangle$. The coalescence of the heavy quarks with the comoving light quarks corresponds to the production of a heavy hadron such as a $\Lambda_{\mathrm{b}}(u d b)$ at small rapidity $\left|y_{\Lambda_{\mathrm{b}}}\right| \simeq \ln x_{b}$, relative to the rapidity of the nucleon in the 645 target. 
6.2. Inclusive production of Higgs boson and intrinsic charm and bottom in proton
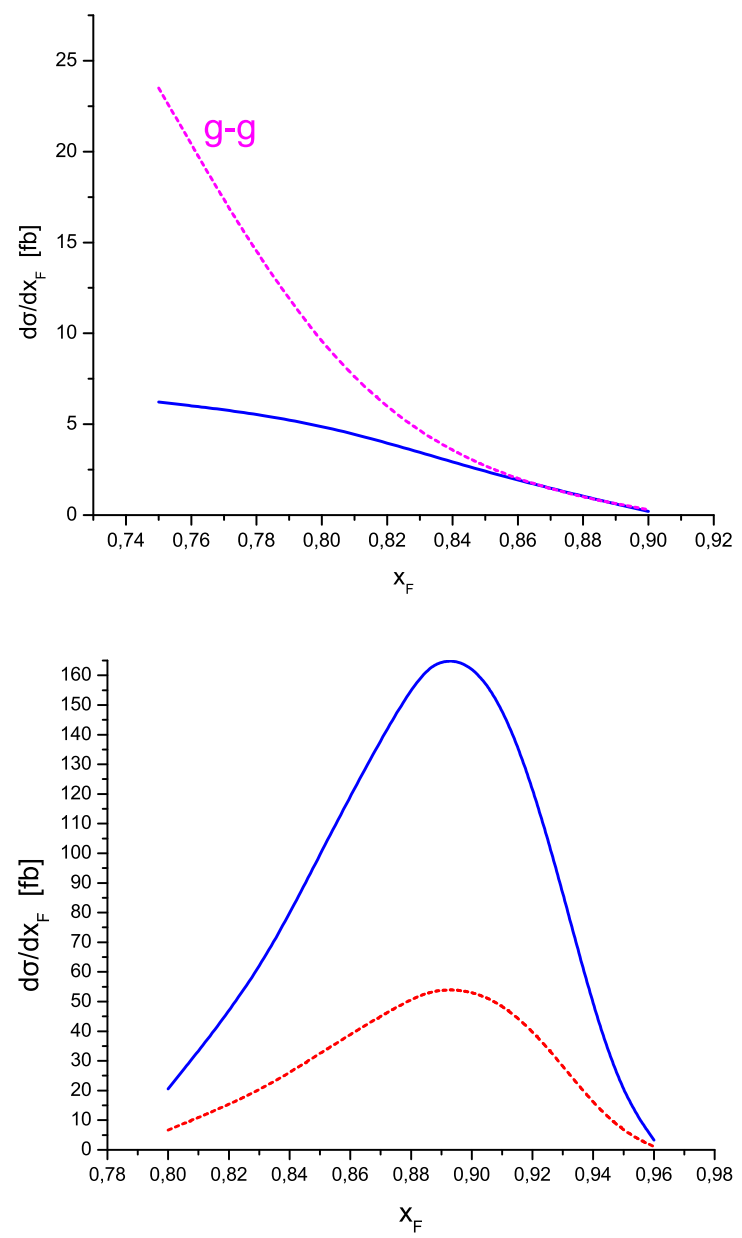

Figure 8: The $x_{\mathrm{F}}$-distribution of the Higgs boson produced in $p p$ collision at the LHC energy $\sqrt{s}=14 \mathrm{TeV} / \mathrm{c}$ due to the non-perturbative intrinsic charm with the probability about $1 \%$ (solid line). The dashed line corresponds to the Higgs boson production from the gluon-gluon fusion (top). The same distribution due to the non-perturbative intrinsic bottom (IB) at the LHC energy $\sqrt{s}=14 \mathrm{TeV} / \mathrm{c}$ (solid line) and the TEVATRON energy $\sqrt{s}=2 \mathrm{TeV}$ (dashed line, bottom) 42 .

The interesting predictions on the possible signal of the intrinsic heavy quark 
(IQ) contributions to the inclusive $x_{\mathrm{F}}$-spectrum of the Higgs bosons produced at the TEVATRON and LHC energies are presented in [42]. In Fig. 8 the contributions of the IC and IB to the $x_{\mathrm{F}}$-distribution of the Higgs boson produced in $p p$ collisions at the collider energies LHC and TEVATRON are presented.

One can see from Fig. 8 that the $x_{\mathrm{F}}$-distribution for the inclusive Higgs boson production coming from the IB contribution is much larger than the one coming ${ }_{55}$ from the IC. It is due to the fact that the Higgs- $Q$ coupling is proportional to the quark mass $m_{\mathrm{Q}}$, therefore the Higgs- $b$ coupling constant is much larger than the coupling constant Higgs- $c$. Fig. 8 also shows that the cross section $\mathrm{d} \sigma / \mathrm{d} x_{\mathrm{F}}$ for the inclusive production of the Standard Model Higgs boson coming from the IB is of order $150 \mathrm{fb}$ at the LHC energy $\sqrt{s}=14 \mathrm{TeV} / \mathrm{c}$, peaking in the region of $x_{\mathrm{F}} \simeq 0.9$. Therefore, the signal of the IB in the differential cross section of the Higgs boson produced in $p p$ collision at high $x_{\mathrm{F}}$ can be tested at the LHC. As is shown in [42, it is much larger than the IC signal.

\subsection{Production of prompt photon and $c$ or b-jet in hard $p p$ collisions}

The investigation of prompt photon and $c(b)$-jet production in $p \bar{p}$ collisions at $\sqrt{s}=1.96 \mathrm{TeV} / \mathrm{c}$ was carried out at the TEVATRON 31 34. In particular, it was observed that the ratio of the experimental spectrum of the prompt photons accompanied by the $c$-jets to the relevant theoretical expectation (based on the conventional PDF, which ignored the IC contribution) increases up to factor of about 3, when $p_{\mathrm{T}}^{\gamma}$ becomes above $110 \mathrm{GeV} / \mathrm{c}$. Furthermore, taking into account the CTEQ66c PDF, which includes the IC contribution obtained within the BHPS model [6, 13] one can reduce this ratio up to 1.5 [37. For the $\gamma+b$-jets $p \bar{p}$-production no enhancement in the $p_{\mathrm{T}}^{\gamma}$-spectrum was observed at the beginning of the experiment [31, 34]. However, in 2012 the DØ collaboration has confirmed observation of such an enhancement 32. It is illustrated in Fig. 9

The dash-dotted lines in Fig.9 (bottom) show the ratio between the NLO calculations of $p_{\mathrm{T}}^{\gamma}$-spectrum obtained within the BHPS model (using the CTEQ66c with the IC probability about $3.5 \%$ ) and the spectrum using the CTEQ6M without the IC contribution. In addition to that the IC signal was visible in the 

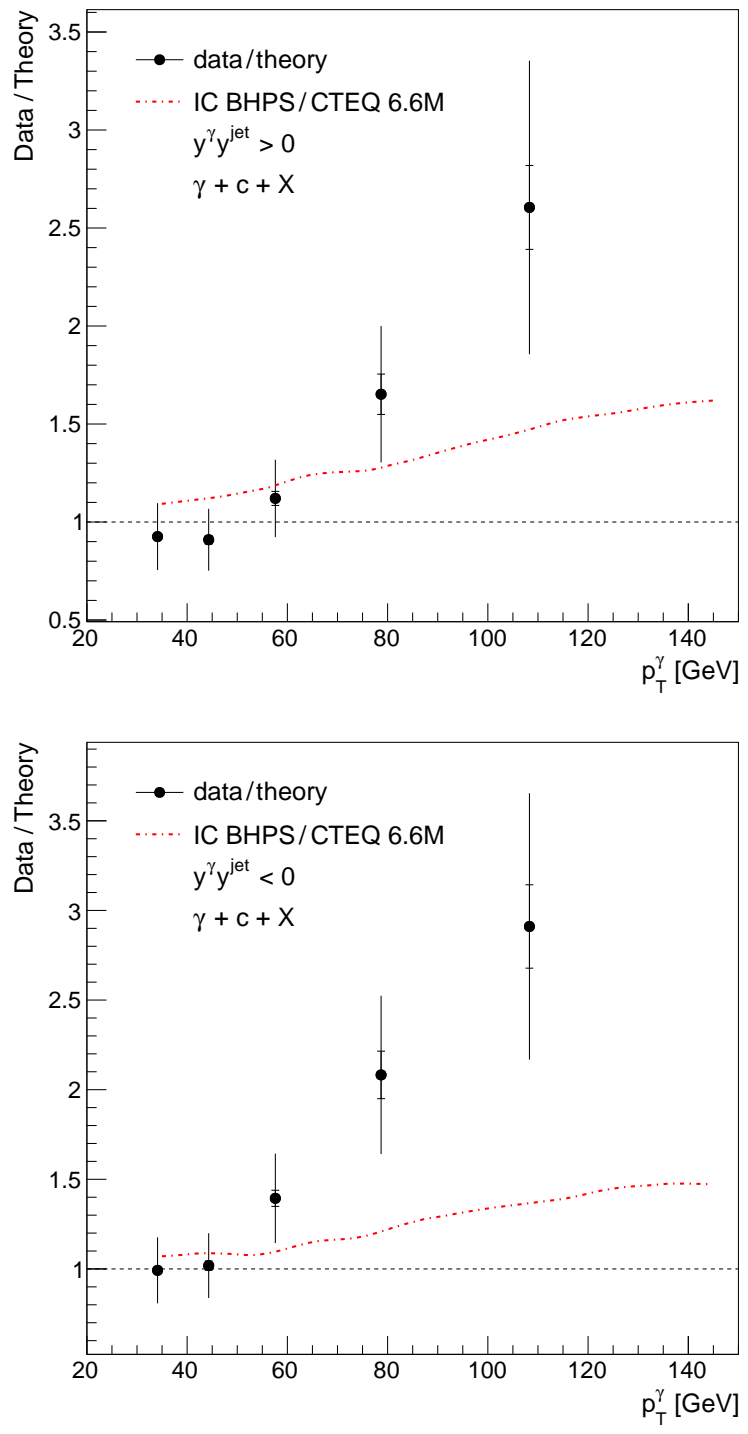

Figure 9: The data-to-theory ratio 31] for the processes $p \bar{p} \rightarrow \gamma+c+X$, when $y^{\gamma} y^{\text {jet }}>0$ (top) and the same ratio, when $y^{\gamma} y^{\text {jet }}<0$ (bottom) at $\sqrt{s}=1.96 \mathrm{TeV} / \mathrm{c}$. The dash-dotted line is the calculation of this ratio using the BHPS IC model with the IC probability about $3.5 \%$.

ratio between the differential cross-sections of the photon and $c$-jet production

in $p \bar{p}$ collision, $\gamma+c$, and $\gamma+b$ production, see Fig. 10. This figure shows that, 
according to the pQCD calculations [37, in the absence of the IC contribution this ratio decreases (solid line), as $p_{\mathrm{T}}^{\gamma}$ grows, while the TEVATRON data show its flat behavior at large $p_{\mathrm{T}} \geq 100 \mathrm{GeV} / \mathrm{c}$. As for the prompt photon production accompanied by the $b$-jet in $p \bar{p}$ annihilation, the TEVATRON data do not show any signal of the intrinsic $b$ contribution, see Fig. 9 (top) [31. It can be due to very small intrinsic bottom probability in a proton, as mentioned above.

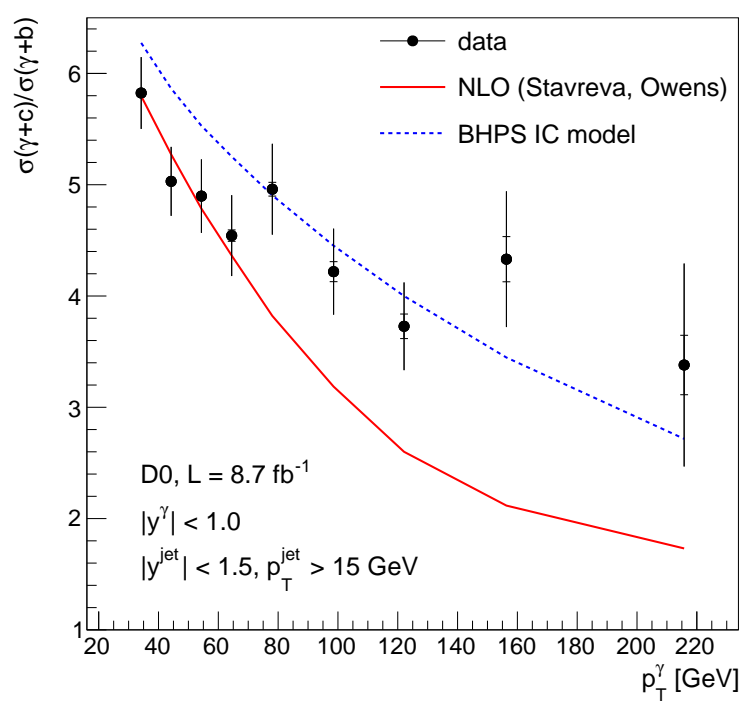

Figure 10: The ratio $\sigma(\gamma+c) / \sigma(\gamma+b)$ as a function of the photon transverse momentum $p_{\mathrm{T}}^{\gamma}$ in $p \bar{p} \rightarrow \gamma+c(b)+X$ process at $\sqrt{s}=1.96 \mathrm{TeV} / \mathrm{c}$, see [33].

This intriguing observation stimulates our interest to look for a similar IC signal in $p p \rightarrow \gamma+c(b)+X$ processes at LHC energies, see [38, 40].

The Examples of Feynman diagrams corresponding to $g g \rightarrow \gamma(Z) Q \bar{Q}$ (a), $q \bar{q} \rightarrow \gamma(Z) Q \bar{Q}(\mathrm{~b}, \mathrm{c})$ and $q Q \rightarrow \gamma(Z) q Q$ (d,e) subprocesses are presented in Fig. 11 .

The diagrams within the NLO QCD are more complicated than Fig. 11.

Let us illustrate qualitatively the kinematical regions where the IC component can contribute significantly to the spectrum of prompt photons produced together with a $c$-jet in $p p$ collisions at the LHC. For simplicity we consider 


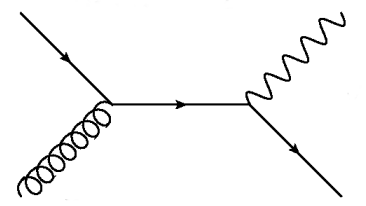

a)

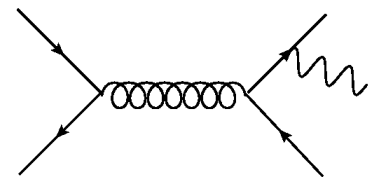

b)

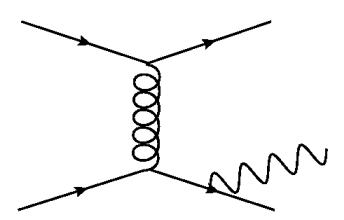

d)

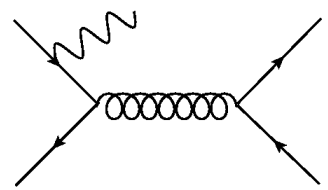

c)

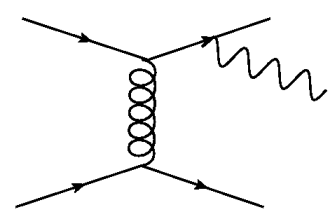

e)

Figure 11: The $\mathcal{O}\left(\alpha \alpha_{\mathrm{s}}\right)$ (a) and $\mathcal{O}\left(\alpha \alpha_{\mathrm{s}}^{2}\right)$ (b) - (e) contributions to the $\gamma(Z)+Q$ production.

only the contribution to the reaction $p p \rightarrow \gamma(Z)+Q+X$ of the diagrams given in Fig. 11 (a). According to 20 at certain values of the transverse momentum of the photon, $p_{\mathrm{T}}^{\gamma}$, and its pseudo-rapidity, $\eta^{\gamma}$, (or rapidity $y^{\gamma}$ ) the momentum fraction of $\gamma$ can be $x_{\mathrm{F}}^{\gamma}>0.1$, therefore the fraction of the initial $c$-quark must also be above 0.1 , where the IC contribution in the proton is enhanced (see Fig. 3. Therefore, one can expect some non-zero IC signal in the $p_{\mathrm{T}}^{\gamma}$ spectrum of the reaction $p p \rightarrow \gamma+c+X$ in this certain region of $p_{\mathrm{T}}^{\gamma}$ and $y^{\gamma}$ [38. A similar IC effect can be visible in the production of the $Z$ or $W$-boson accompanied by $c$ or $b$-jets in $p p$ collisions. In 39,40 , the theoretical predictions about the possible observation of the IC signal in the $p_{\mathrm{T}}$-spectra of $Z$ or $W$-bosons accompanied by the $c$ or $b$-jets respectively are presented. These processes will be considered later. First, let us discuss a possible search for the IC signal in the production of prompt photons accompanied by $c$-jet in $p p$ collision at LHC energies. 
In Fig. 12 the differential cross-section $\mathrm{d} \sigma / \mathrm{d} p_{\mathrm{T}}^{\gamma}$ calculated at NLO within the collinear QCD massless quark approximation as described in [37] is presented as a function of the transverse momentum of the prompt photon [38. The following requirements are applied: $p_{\mathrm{T}}^{\gamma}>45 \mathrm{GeV} / \mathrm{c}, p_{\mathrm{T}}^{\mathrm{c}}>20 \mathrm{GeV} / \mathrm{c}$ with the $c$-jet pseudorapidity in the interval $\left|y_{c}\right| \leq 2.4$ and the photon pseudorapidity in the interval $1.52<\left|y_{\gamma}\right|<2.37$ (forward region). The solid line represents the differential cross-section calculated with the radiatively generated charm PDF (CTEQ66), the dash-dotted line uses as input the sea-like PDF (CTEQ66c4) and the dashed line the BHPS PDF (CTEQ66c2). In the lower half of Fig. 12 the above distributions normalized to the distribution acquired using the CTEQ66 $\mathrm{PDF}$ and $\mu_{\mathrm{r}}=\mu_{\mathrm{f}}=\mu_{\mathrm{F}}=p_{\mathrm{T}}^{\gamma}$, are presented. The shaded yellow region, represents the scale dependence. Clearly the difference between the spectrum using the BHPS IC PDF and the one using the radiatively generated PDF increases as $p_{\mathrm{T}}^{\gamma}$ grows.

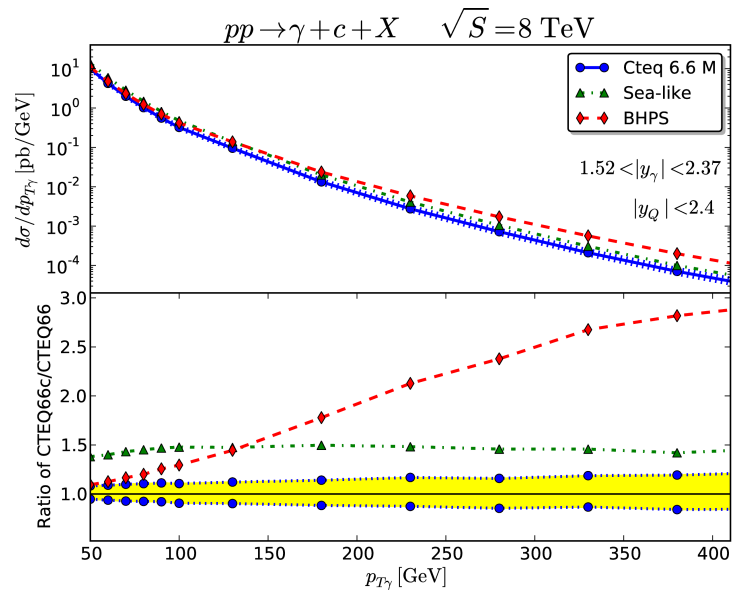

Figure 12: The $\mathrm{d} \sigma / \mathrm{d} p_{\mathrm{T}}^{\gamma}$ distribution versus the transverse momentum of the photon for the process $p p \rightarrow \gamma+c+X$ at $\sqrt{s}=8 \mathrm{TeV} / \mathrm{c}$ using CTEQ6.6M (solid blue line), BHPS CTEQ6c2 (dashed red line) and sea-like CTEQ6c4 (dash-dotted green line), for forward photon rapidity $1.52<\left|y_{\gamma}\right|<2.37$. The ratio of these spectra with respect to the CTEQ6.6M (solid blue line) distributions (bottom). The calculation was done within the NLO QCD approximation.

Therefore, Fig. 12 shows that the IC signal could be visible at the LHC 
energies with both the ATLAS and CMS detector in the process $p p \rightarrow \gamma+c+X$ when $p_{\mathrm{T}}^{\gamma} \simeq 150 \mathrm{GeV} / \mathrm{c}$. In the region described above the IC signal dominates over the all non-intrinsic charm background with significance at a level of a factor of 2 (in fact $170 \%$ ).

\subsection{Production of $\gamma(Z)+c(b)$-jet within the $k_{\mathrm{T}}$-factorization and the $M C F M$}

The $k_{\mathrm{T}}$-factorization approach [86 88] is based on the small- $x$ BalitskyFadin-Kuraev-Lipatov (BFKL) 89 91] gluon dynamics and provides solid theoretical ground for the effects of the initial gluon radiation and the intrinsic parton transverse momentum 1 Our main motivation to use the $k_{\mathrm{T}}$-factorization formalism here is that its predictions for the associated $\gamma+Q$ production better agree with the TEVATRON data compared to the NLO pQCD (see [32, 35]). The consideration is mainly based on the $\mathcal{O}\left(\alpha \alpha_{\mathrm{s}}\right)$ off-shell (depending on the transverse momenta of initial quarks and gluons) quark-gluon Compton-like scattering subprocess, see Fig. 11 (a). Within this approach the transverse momentum dependent (TMD) parton densities include many high order corrections, while the partonic amplitudes are calculated within the leading order (LO) of QCD. The off-shell quark-gluon Compton scattering amplitude is calculated within the reggeized parton approach 95 99. based on the effective action formalism [100, 101, which ensures the gauge invariance of the obtained amplitudes despite the off-shell initial quarks and gluons ${ }^{2}$ The TMD parton densities are calculated using the Kimber-Martin-Ryskin (KMR) approach, currently developed within the NLO [103. This approach is the formalism to construct the TMD quark and gluon densities from the known conventional parton distributions. The key assumption is that the $k_{\mathrm{T}}$ dependence appears at the last evolution step, so that the DGLAP evolution can be used up to this step. Numerically, for the input we used parton densities derived in Section 2. Other details of these calculations are explained in [102.

\footnotetext{
${ }^{1} \mathrm{~A}$ detailed description of the $k_{\mathrm{T}}$-factorization approach can be found, for example, in reviews 9294

${ }^{2}$ Here we use the expressions derived earlier 102 .
} 
To improve the $k_{\mathrm{T}}$-factorization predictions at high transverse momenta, we take into account some $\mathcal{O}\left(\alpha \alpha_{\mathrm{s}}^{2}\right)$ contributions, namely $q \bar{q} \rightarrow V Q \bar{Q}$ and $q Q \rightarrow V q Q$ ones, where $V$ denotes the photon or the $Z$ boson, see Fig. $11((\mathrm{~b})-(\mathrm{e}))$. These contributions are significant at large $x$ and therefore can be calculated in the usual collinear QCD factorization scheme. Thus, we rely on the combination of two techniques that are most suitable.

Let us present the results of our calculations. First of all we describe our numerical input. Following to [103, we set the charmed and bottom quark masses $m_{\mathrm{c}}=1.4 \mathrm{GeV} / \mathrm{c}, m_{\mathrm{b}}=4.75 \mathrm{GeV} / \mathrm{c}$, the $Z$-boson mass $m_{\mathrm{Z}}=91.1876 \mathrm{GeV} / \mathrm{c}$, and $\sin ^{2} \theta_{\mathrm{W}}=0.23122$. The chosen factorization and renormalization scales are $\mu_{\mathrm{R}}=\mu_{\mathrm{F}}=\xi p_{\mathrm{T}}$ or $\mu_{\mathrm{R}}=\mu_{\mathrm{F}}=\xi m_{\mathrm{T}}$, where $p_{\mathrm{T}}$ is the produced photon transverse momentum and $m_{\mathrm{T}}$ is the $Z$ boson transverse mass. As usual, we vary the non-physical parameter $\xi$ between $1 / 2$ and 2 about the default value $\xi=1$ in order to estimate the scale uncertainties of our calculations. We employ the two-loop formula for the strong coupling constant with active quark flavors $n_{\mathrm{f}}=5$ at $\Lambda_{\mathrm{QCD}}=226.2 \mathrm{MeV} / \mathrm{c}$ and use the running QED coupling constant over a wide region of transverse momenta. The multidimensional integration in the $k_{\mathrm{T}}$-factorization calculations was performed by means of the Monte Carlo technique, using the VEGAS routine [104].

In our calculations we also follow the conclusion obtained in our papers [38, 39 that the IC signal in the hard processes discussed here can be detected at ATLAS or CMS of the LHC in the forward rapidity region $1.5<|\eta|<2.4$ and $p_{\mathrm{T}}>50 \mathrm{GeV} / \mathrm{c}$. Additionally, we require $|\eta(Q)|<2.4$ and $p_{\mathrm{T}}(Q)>25 \mathrm{GeV} / \mathrm{c}$, where $\eta(Q)$ and $p_{\mathrm{T}}(Q)$ are the pseudo-rapidity and transverse momentum of the heavy quark jet in a final state, as was done in [38, 39].

The results of our calculations are shown in Figs. 13-21. The transverse momentum distributions of photons and $Z$ bosons accompanied by the $c$ and $b$ quarks are presented in Figs. 13,14 and 15 at the different IC probability $w$ (namely, $w=0 \%, w=2 \%$ and $w=3.5 \%$ ) at $\sqrt{s}=8$ and $13 \mathrm{TeV} / \mathrm{c}$.

One can see in Figs. 14 and 15 that the MCFM and $k_{\mathrm{T}}$-factorization predictions for $Z+Q$ production are very similar in the whole $p_{\mathrm{T}}$ region, therefore 

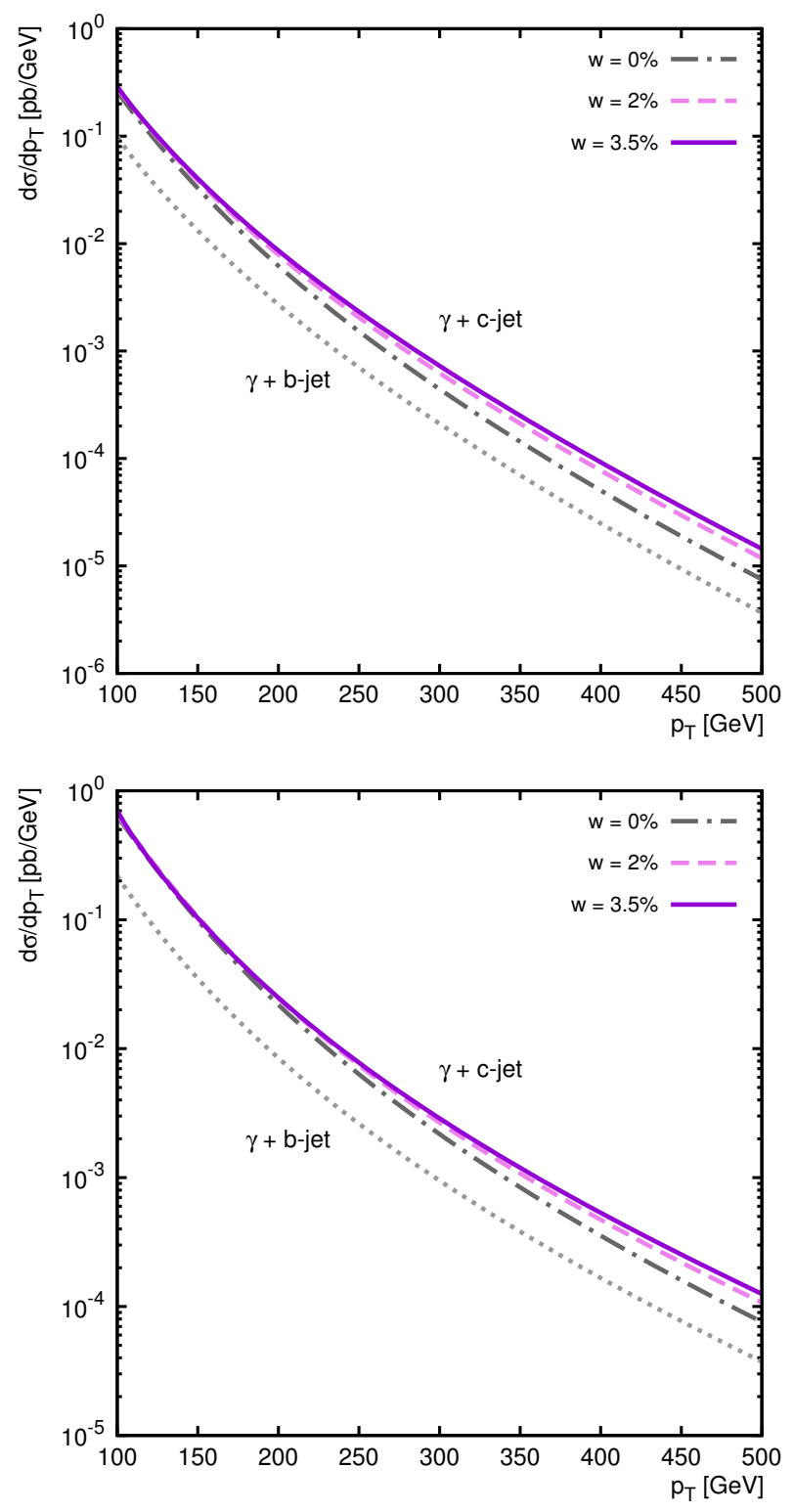

Figure 13: The cross-sections of the associated $\gamma+c$ and $\gamma+b$ production in the $p p$ collision calculated as a function of the photon transverse momentum $p_{\mathrm{T}}$ at $\sqrt{s}=8 \mathrm{TeV} / \mathrm{c}$ (top) and $\sqrt{s}=13 \mathrm{TeV} / \mathrm{c}$ (bottom) within the $k_{\mathrm{T}}$-factorization approach. The kinematical conditions are described in the text. 

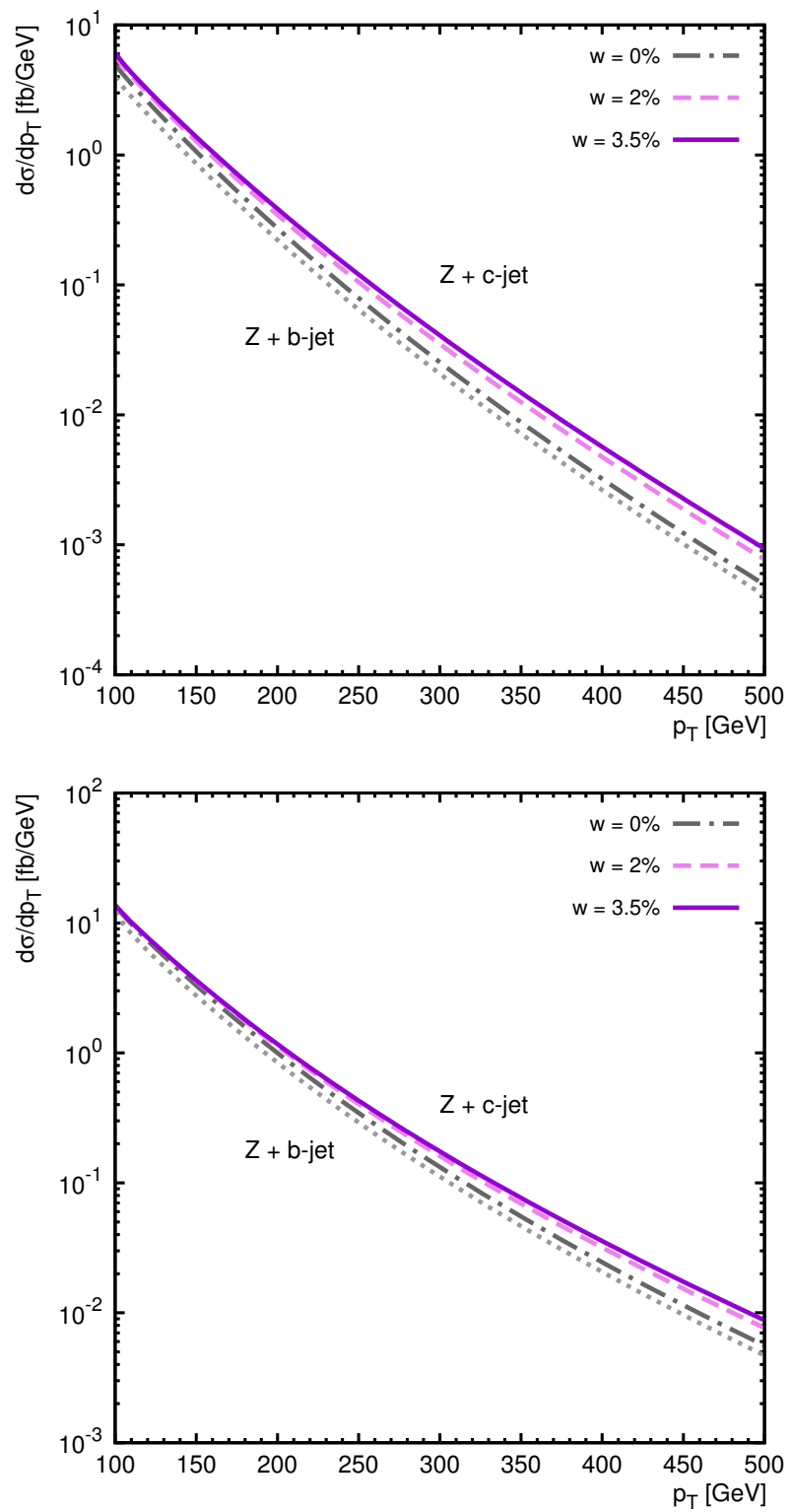

Figure 14: The cross-sections of the associated $Z+c$ and $Z+b$ production in the $p p$ collision calculated as a function of the $Z$ boson transverse momentum $p_{\mathrm{T}}$ at $\sqrt{s}=8 \mathrm{TeV} / \mathrm{c}$ (top) and $\sqrt{s}=13 \mathrm{TeV} / \mathrm{c}$ (bottom) within the $k_{\mathrm{T}}$-factorization approach. The kinematical conditions are described in the text. 

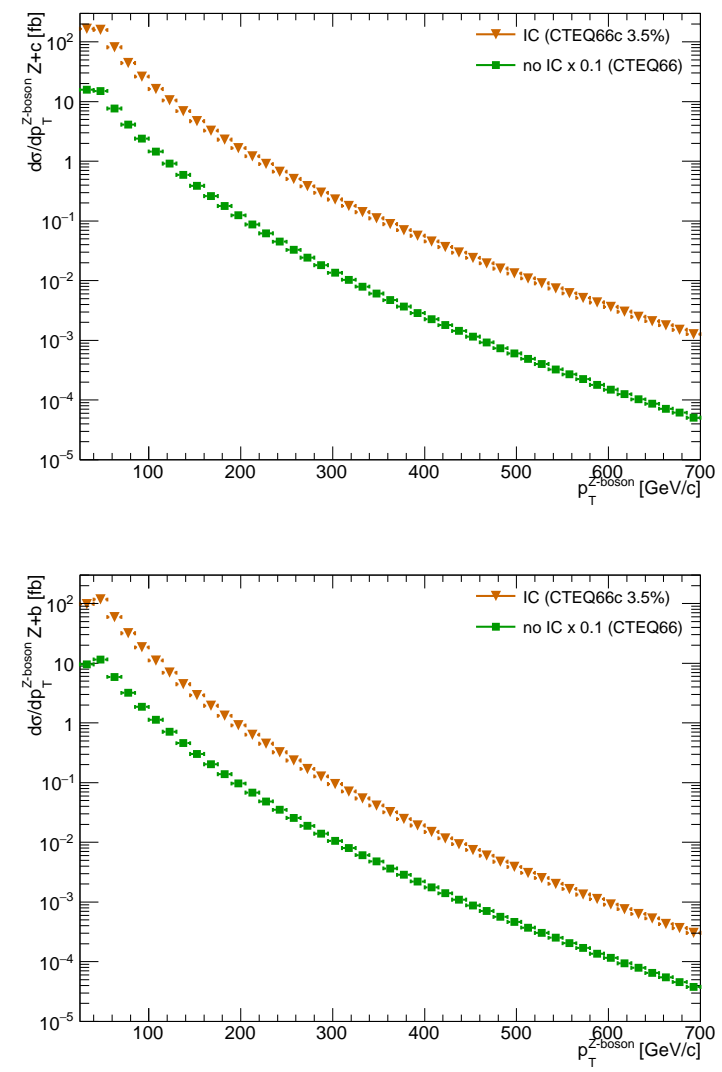

Figure 15: The cross-sections of the associated $Z+c$ (top) and $Z+b$ (bottom) production in $p p$ collision calculated as a function of the $Z$ boson transverse momentum $p_{\mathrm{T}}$ at $\sqrt{s}=13 \mathrm{TeV} / \mathrm{c}$ within the MCFM routine.

below we will present the observables calculated within the $k_{\mathrm{T}}$-factorization approach only. The coincidence of these two calculations is due to effective allowance for the high-order corrections within the $k_{\mathrm{T}}$-factorization formalism (see, for example, 92 94 for more information). Both types of calculations predict a significant enhancement of $p_{\mathrm{T}}$ distributions due to the IC terms at $p_{\mathrm{T}} \geq 100 \mathrm{GeV} / \mathrm{c}$, which is in agreement with the previous studies [38, 39, 64].

The $p_{\mathrm{T}}$ spectrum ratios $\sigma(\gamma+c) / \sigma(\gamma+b)$ and $\sigma(Z+c) / \sigma(Z+b)$ versus $p_{\mathrm{T}}$ at different $w$ are presented in Figs. 16 and 17 . One can see that in the absence 
and decreases down to 2 at $p_{\mathrm{T}} \sim 500 \mathrm{GeV} / \mathrm{c}$. This behavior is the same for both energies $\sqrt{s}=8 \mathrm{TeV} / \mathrm{c}$ and $\sqrt{s}=13 \mathrm{TeV} / \mathrm{c}$.

If one takes into account the IC contributions, this ratio becomes approximately flat at $w=2 \%$ or even increasing up to about 4 at $w=3.5 \%$. It $b) \sim 3.5-4.5$ measured in the $p \bar{p}$ collisions at $110<p_{\mathrm{T}}<300 \mathrm{GeV} / \mathrm{c}$ and $\sqrt{s}=1.96 \mathrm{TeV} / \mathrm{c}$. However, this agreement cannot be treated as the IC indication due to huge experimental uncertainties (about $50 \%$ ) and rather different kinematical conditions. If the IC contribution is included, the ratio ${ }_{800} \sigma(Z+c) / \sigma(Z+b)$ also increases by a factor about 2 at $w=3.5 \%$, when the $Z$ boson transverse momentum grows from $100 \mathrm{GeV} / \mathrm{c}$ to $500 \mathrm{GeV} / \mathrm{c}$ (see Fig. 17). In the absence of the IC terms this ratio slowly decreases.

One can consider other observables which could be useful to detect the IC signal, the cross-sections discussed above but integrated over $p_{\mathrm{T}}>p_{\mathrm{T}}^{\mathrm{min}}$, where $p_{\mathrm{T}}^{\min } \geq 100 \mathrm{GeV} / \mathrm{c}$, and their ratios. Our predictions for such integrated crosssections versus the IC probability $w$ at $p_{\mathrm{T}}^{\min }=100,200$ and $300 \mathrm{GeV} / \mathrm{c}$ for $\sqrt{s}=8 \mathrm{TeV} / \mathrm{c}$ and $p_{\mathrm{T}}^{\min }=200,300$ and $400 \mathrm{GeV} / \mathrm{c}$ for $\sqrt{s}=13 \mathrm{TeV} / \mathrm{c}$ are shown in Figs. 18, 19 and Figs. 20, 21.

All the $p_{\mathrm{T}}$-spectra have a significant scale uncertainty as is shown in [39]. According to [39], the ratio between the cross-sections for the $Z+Q$ and $W+Q$ production in the $p p$ collision is less sensitive to the scale variation calculated within the MCFM. Nevertheless, the uncertainty in this ratio at large $p_{\mathrm{T}}>250 \mathrm{GeV} / \mathrm{c}$ is about $40-50 \%$. In the present paper we check these results for the ratios $\sigma(\gamma+c) / \sigma(\gamma+b)$ and $\sigma(Z+c) / \sigma(Z+b)$. In Figs. 18, 19 and Figs. 20, 21 we present these ratios versus the IC probability $w$ calculated at different scales, when the cross-sections of $\gamma(Z)+Q$ production are integrated within the different intervals of transverse momentum. One can see a very small QCD scale uncertainty, especially at $\sqrt{s}=13 \mathrm{TeV} / \mathrm{c}$ (bottom right), which is less than $1 \%$. In contrast, the scale uncertainty for the integrated $\gamma(Z)+Q$ cross-sections (see Figs. 18, 19 820 and Figs. 20, 21] is significant and amounts to about $30-40 \%$. The sizable 

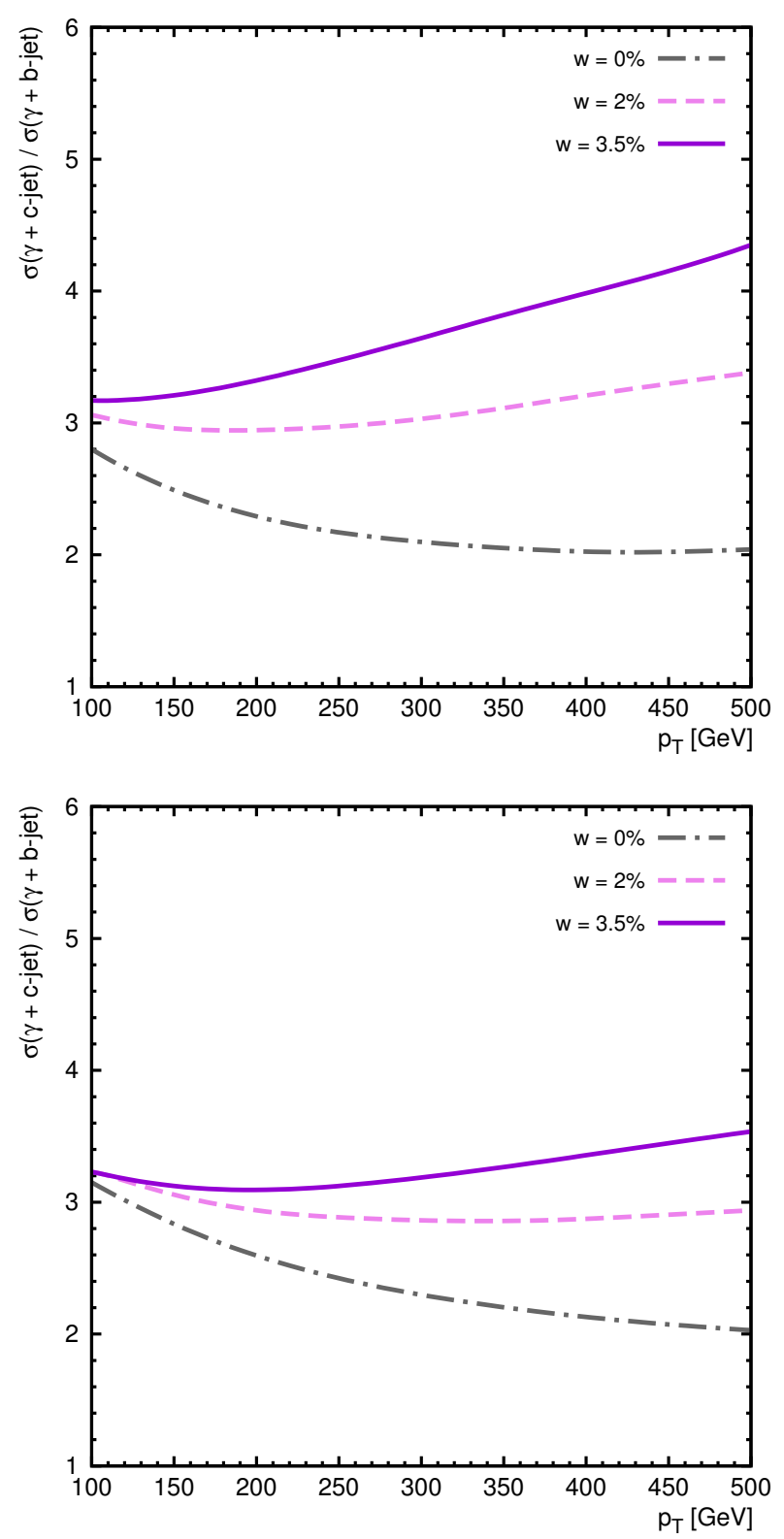

Figure 16: The cross-section ratio of the $\gamma+c$ production to the $\gamma+b$ one in the $p p$ collision calculated as a function of the photon transverse momentum $p_{\mathrm{T}}$ at $\sqrt{s}=8 \mathrm{TeV} / \mathrm{c}$ (top) and $\sqrt{s}=13 \mathrm{TeV} / \mathrm{c}$ (bottom) within the $k_{\mathrm{T}}$-factorization approach. 

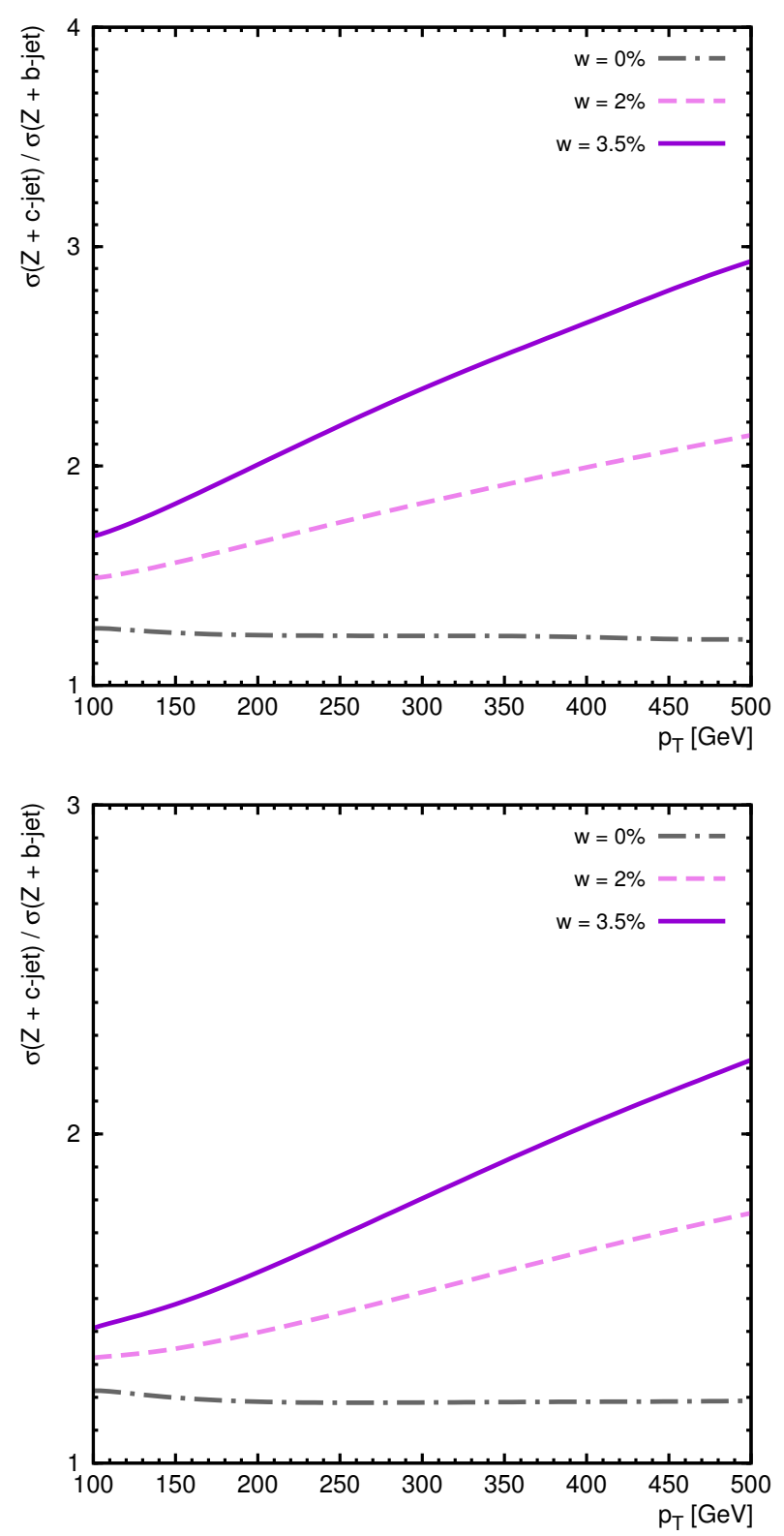

Figure 17: The cross-section ratio of the $Z+c$ production to the $Z+b$ one in the $p p$ collision calculated as a function of the $Z$ boson transverse momentum $p_{\mathrm{T}}$ at $\sqrt{s}=8 \mathrm{TeV} / \mathrm{c}$ (top) and $\sqrt{s}=13 \mathrm{TeV} / \mathrm{c}$ (bottom) within the $k_{\mathrm{T}}$-factorization approach. 

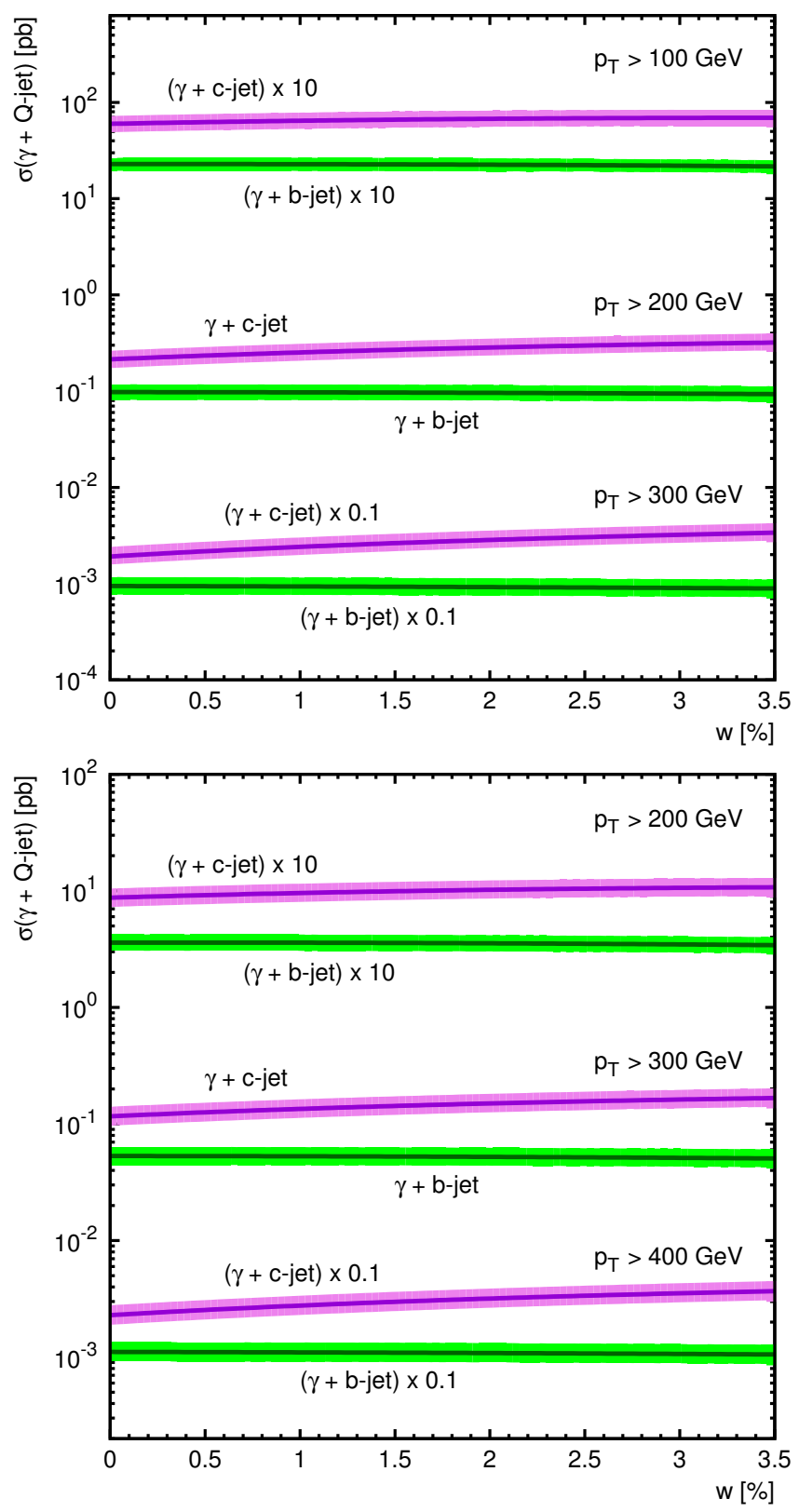

Figure 18: The cross-sections of the associated $\gamma+c$ and $\gamma+b$ production in the $p p$ collision as a function of $w$ integrated over the photon transverse momenta $p_{\mathrm{T}}>p_{\mathrm{T}}^{\min }$ for different $p_{\mathrm{T}}^{\min }$ at $\sqrt{s}=8 \mathrm{TeV} / \mathrm{c}($ top) and $\sqrt{s}=13 \mathrm{TeV} / \mathrm{c}$ (bottom). 

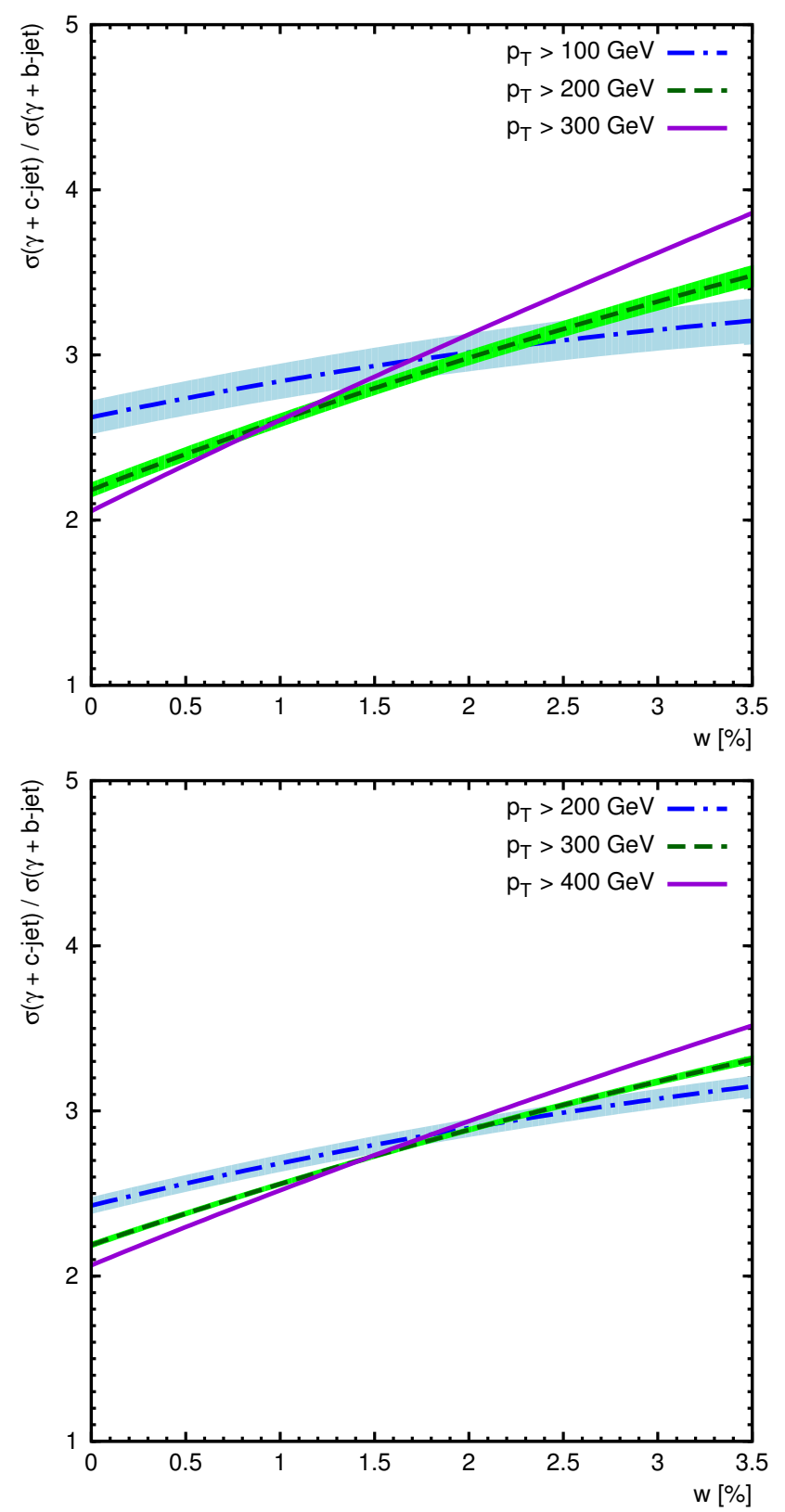

Figure 19: The corresponding ratios of these cross-sections. The calculations were done using the $k_{\mathrm{T}}$-factorization approach. The bands correspond to the usual scale variation as it is described in the text. 

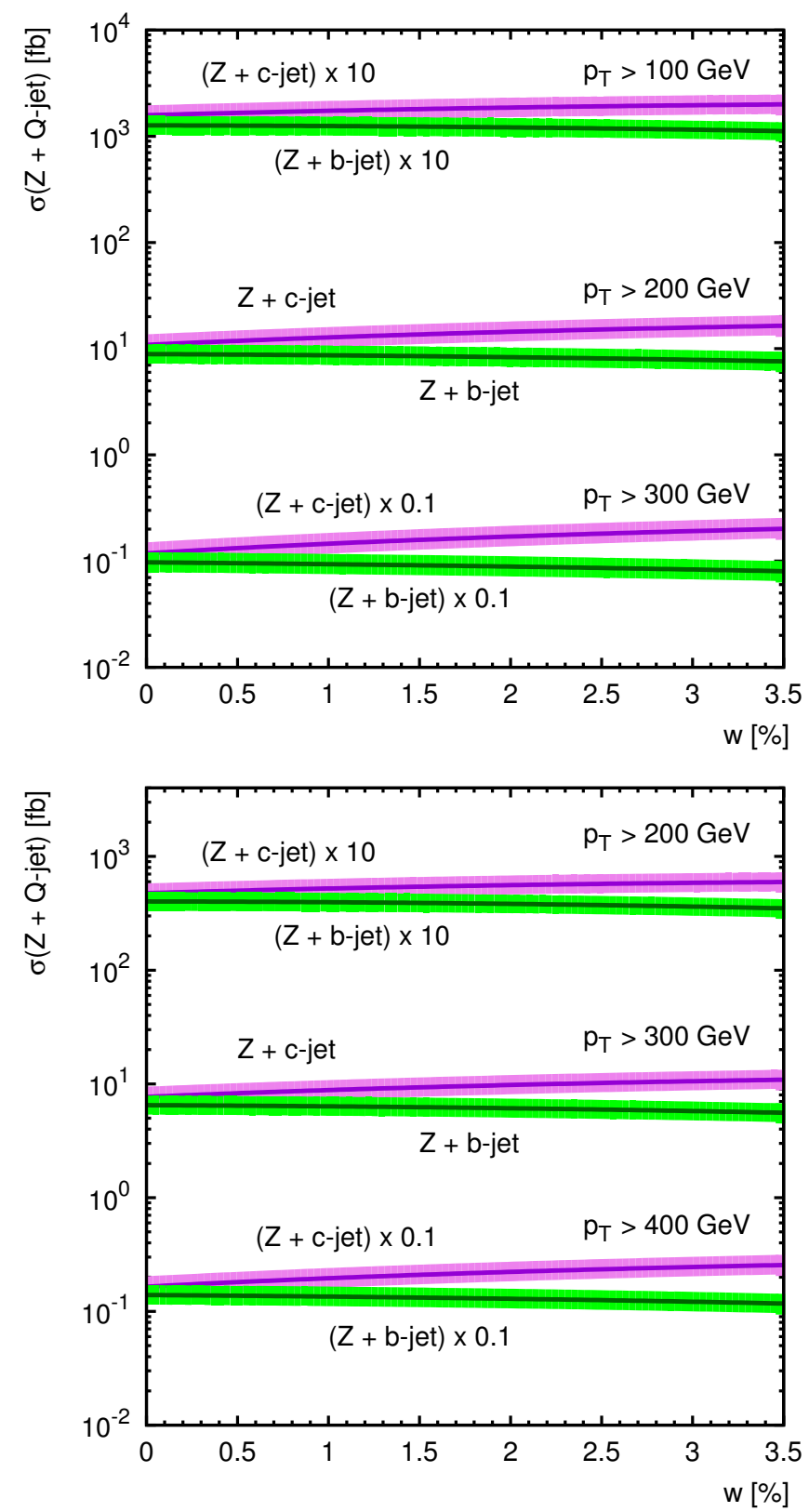

Figure 20: The cross-sections of the associated $Z+c$ and $Z+b$ production in the $p p$ collision as a function of $w$ integrated over the $Z$ boson transverse momenta $p_{\mathrm{T}}>p_{\mathrm{T}}^{\min }$ for different $p_{\mathrm{T}}^{\min }$ at $\sqrt{s}=8 \mathrm{TeV} / \mathrm{c}$ (top) and $\sqrt{s}=13 \mathrm{TeV} / \mathrm{c}$ (bottom). The kinematical conditions are described in the text. 

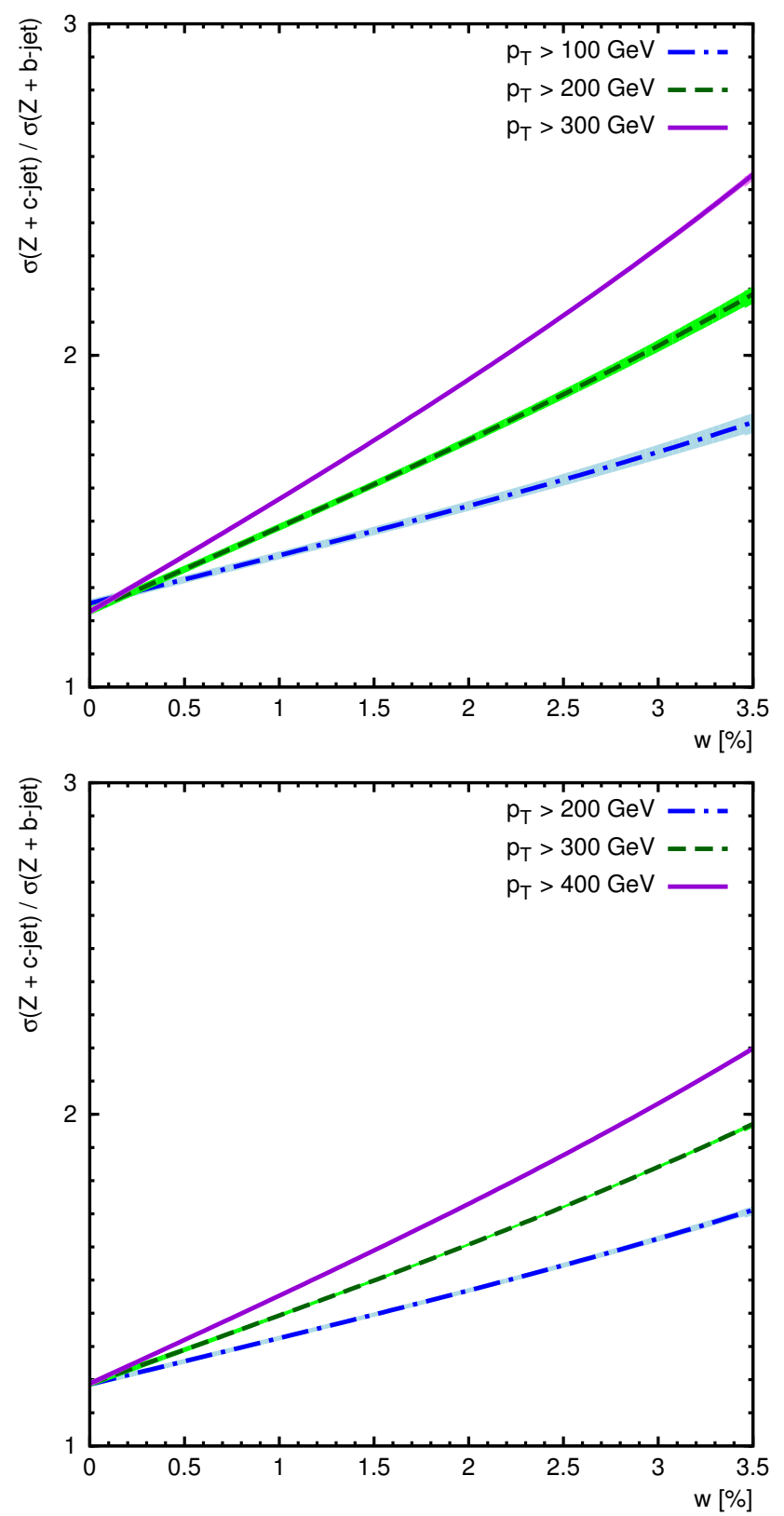

Figure 21: The corresponding ratios of these cross-sections. The calculations were done using the $k_{\mathrm{T}}$-factorization approach. The bands correspond to the usual scale variation as it is described in the text. 
difference between the scale uncertainties for the ratios $\sigma(Z+Q) / \sigma(W+Q)$ and $\sigma(Z+c) / \sigma(Z+b)$ is due to the different matrix elements for the $Z+Q$ and $W+Q$ production in $p p$ collisions, while the matrix elements for the $Z+c$ and $Z+b$ production are the same.

825 It is important that the calculated ratios $\sigma(\gamma+c) / \sigma(\gamma+b)$ and $\sigma(Z+c) / \sigma(Z+$ $b$ ) can be used to determine the IC probability $w$ from the future LHC data. Moreover, these ratios are practically independent of the uncertainties of our calculations: actually, the curves corresponding to the usual scale variations as described above coincide with each other (see Figs. 19 and 21. bottom).

830 Therefore, we can recommend these observables as a test for the hypothesis of the IC component inside the proton.

\subsection{Prompt photon and open charm production}

In Fig. 22 the differential cross-sections of prompt photons accompanied by the $D^{*}$-mesons calculated as a function of produced photon transverse momenta 835 at $\sqrt{s}=8 \mathrm{TeV} / \mathrm{c}$ (top) and $13 \mathrm{TeV} / \mathrm{c}$ (bottom) are presented with and without the IC contribution, and the ratios of these spectra are shown in Fig. 23. The calculations were performed using the $k_{\mathrm{T}}$-factorization approach and the kinematical requirements applied are the same as above. We produce $D^{*}$ mesons from charmed quarks using the Peterson fragmentation function with a shape 840 parameter $\epsilon_{c}=0.06$, and the branching fraction $f\left(c \rightarrow D^{*}\right)$ is equal to 0.255 . In Fig. 23 the ratio of the $p_{\mathrm{T}}$-spectra of the photons accompanied by the $D^{*}$ mesons in $p p$ collisions with the intrinsic $3.5 \%$ charm contribution and without it at $\sqrt{s}=8 \mathrm{TeV} / \mathrm{c}$ (top) and $\sqrt{s}=13 \mathrm{TeV} / \mathrm{c}$ (bottom). One can see that the IC signal in the $\gamma+D^{*}$ cross-section is practically the same as in the case of ${ }_{845} \gamma+c$-jet production.

\section{6. $W(Z)$-boson and $b(c)$-jet production}

Let us analyze another hard processes of the production of vector boson accompanied by the $b$ and $c$ jets in $p p$ collision, which can give us also information on the intrinsic charm in proton [39. The LO QCD diagram for the process 

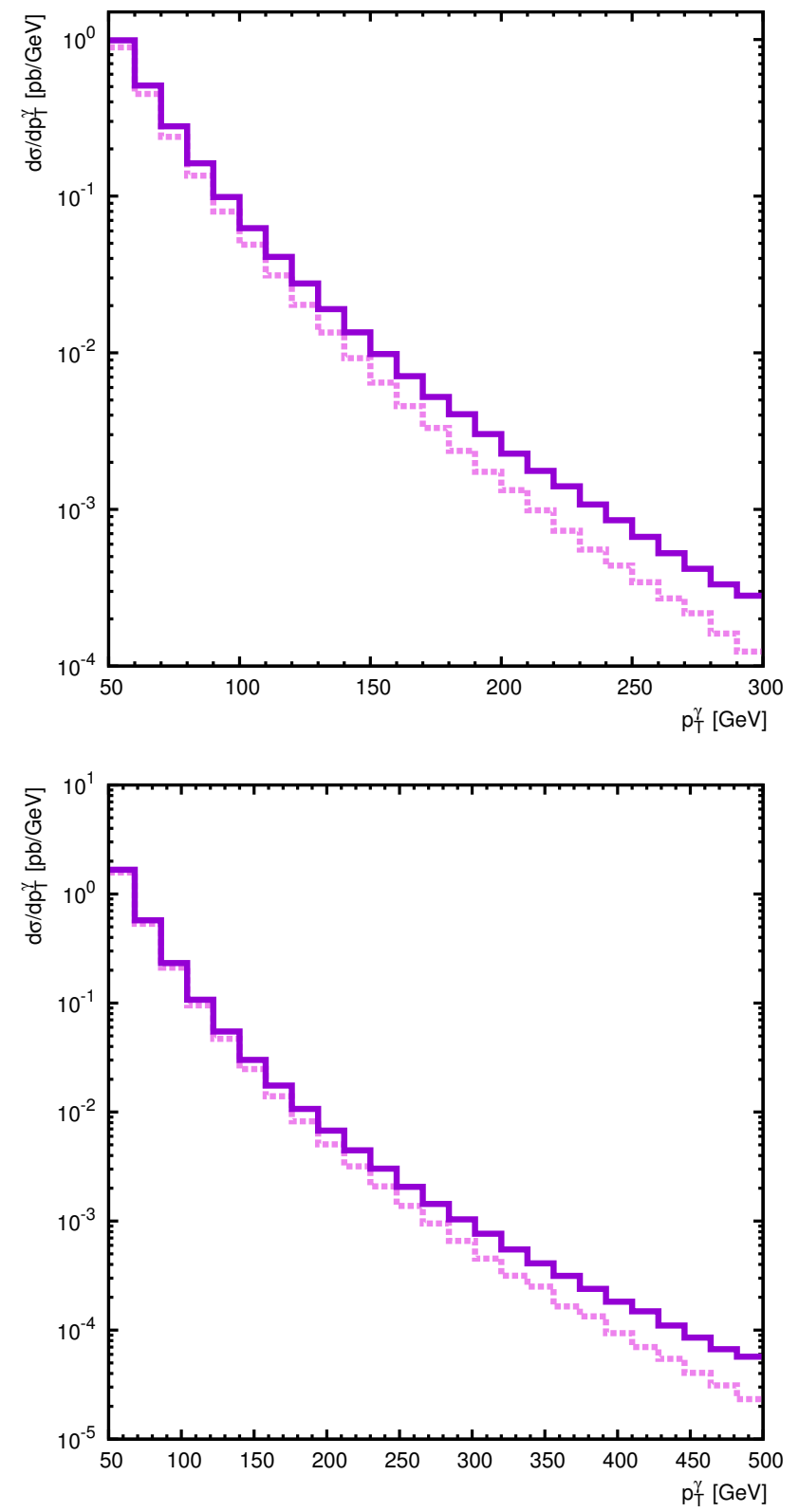

Figure 22: The $p_{\mathrm{T}}$-spectra of the photons accompanied by the $D^{*}$-mesons in $p p$ collisions with the intrinsic $3.5 \%$ charm contribution and without it at $\sqrt{s}=8 \mathrm{TeV} / \mathrm{c}$ (top) and $\sqrt{s}=13 \mathrm{TeV} / \mathrm{c}$ (bottom). Both plots correspond to $1.5<\left|y^{\gamma}\right|<2.4,\left|y^{\text {jet }}\right|<2.4$. 

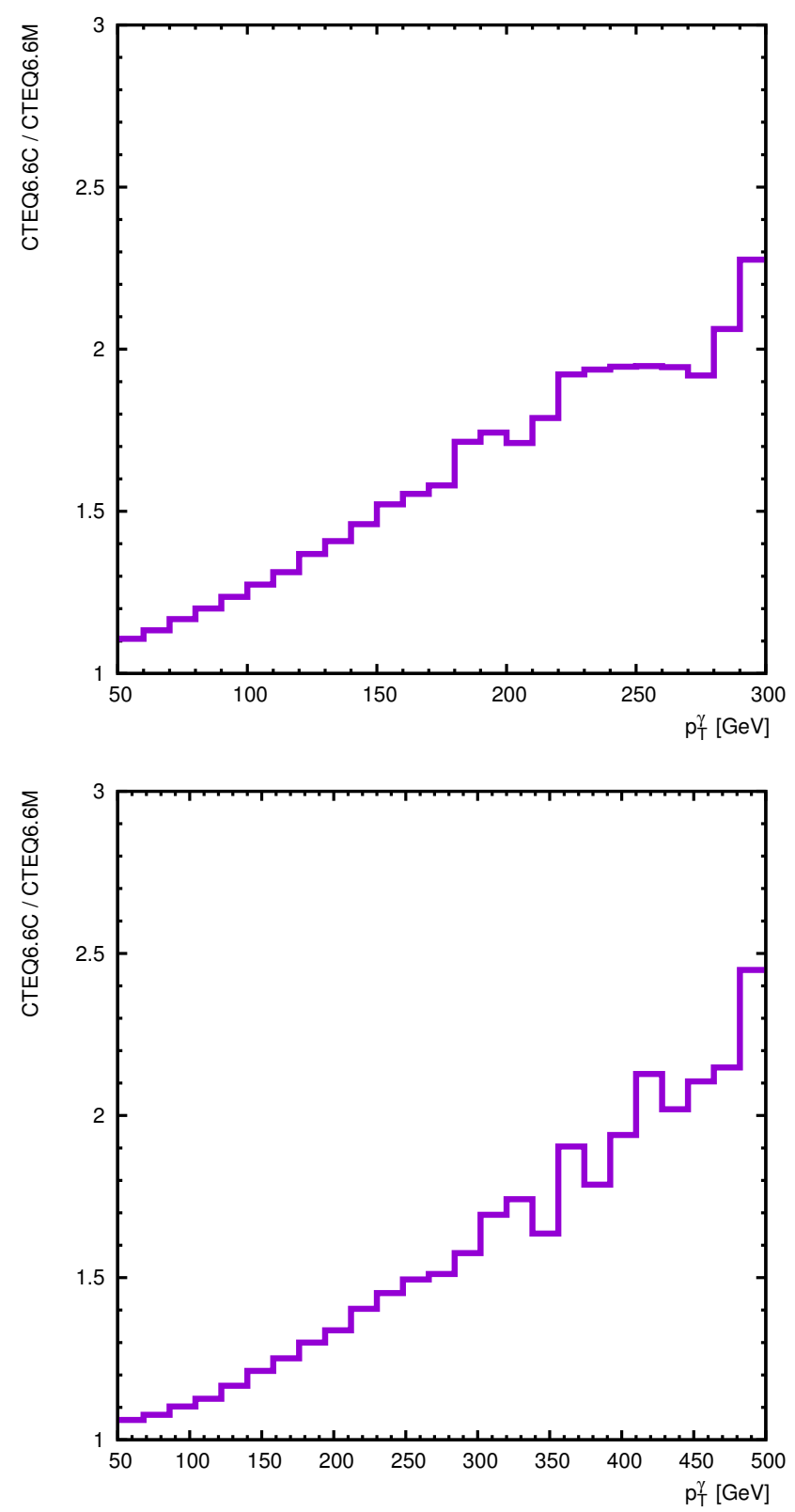

Figure 23: The ratios of the $p_{\mathrm{T}}$-spectra of the photons accompanied by the $D^{*}$-mesons in $p p$ collisions with the intrinsic $3.5 \%$ charm contribution and without it at $\sqrt{s}=8 \mathrm{TeV} / \mathrm{c}$ (top) and $\sqrt{s}=13 \mathrm{TeV} / \mathrm{c}$ (bottom). Both plots correspond to $1.5<\left|y^{\gamma}\right|<2.4,\left|y^{\text {jet }}\right|<2.4$. 
$Q_{\mathrm{f}}\left(\bar{Q}_{\mathrm{f}}\right)+g \rightarrow Z^{0}+Q_{\mathrm{f}}\left(\bar{Q}_{\mathrm{f}}\right)$ is presented in Fig. 24 . These hard subprocesses can give the main contribution to the reaction $p p \rightarrow Z^{0}\left(\rightarrow l^{+}+l^{-}\right)+Q_{\mathrm{f}}\left(\bar{Q}_{\mathrm{f}}\right)-j e t+$ $X$, which could also give us information on the IC contribution in the proton.

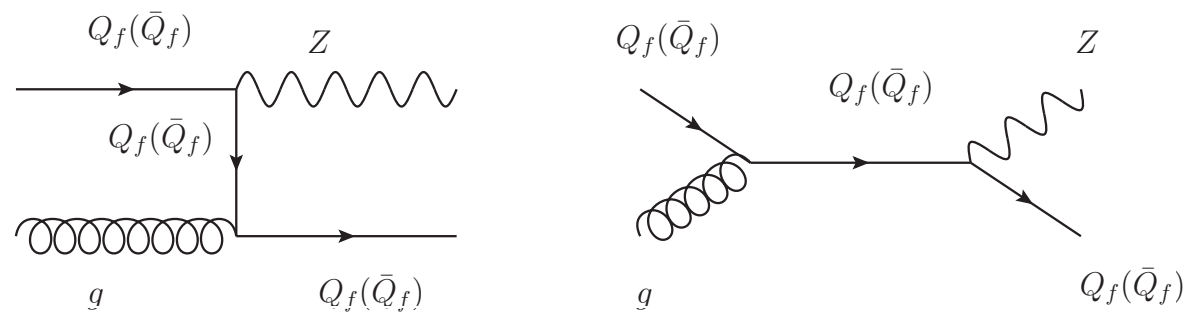

Figure 24: LO Feynman diagrams for the process $Q_{f}\left(\bar{Q}_{f}\right) g \rightarrow Z Q_{f}\left(\bar{Q}_{f}\right)$, where $Q_{f}=c, b$.

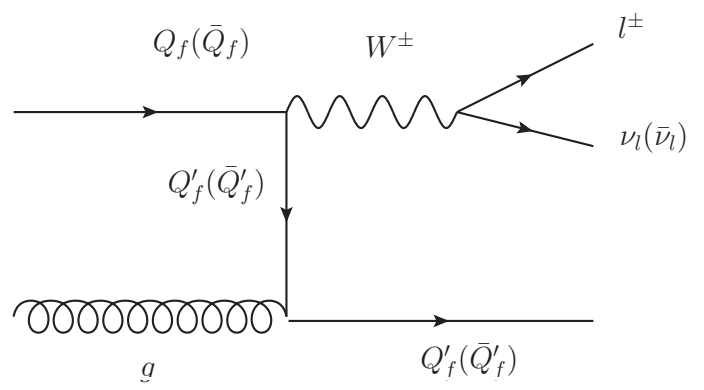

Figure 25: Example of an LO Feynman diagram for the process $Q_{\mathrm{f}}\left(\bar{Q}_{\mathrm{f}}\right) g \rightarrow W^{ \pm} Q_{\mathrm{f}}^{\prime}\left(\bar{Q}_{\mathrm{f}}^{\prime}\right)$, where $Q_{\mathrm{f}}=c, b$ and $Q_{\mathrm{f}}^{\prime}=b, c$ respectively.

The LO QCD diagram for the process $Q_{\mathrm{f}}\left(\bar{Q}_{\mathrm{f}}\right)+g \rightarrow W^{ \pm}+Q_{\mathrm{f}}^{\prime}\left(\bar{Q}_{\mathrm{f}}^{\prime}\right)$ is presented in Fig. 25, where $Q_{\mathrm{f}}=c, b$ and $Q_{\mathrm{f}}^{\prime}=b, c$. These hard subprocesses can give the main contribution to the reaction $p p \rightarrow W^{ \pm}\left(\rightarrow l^{+}+\nu\right)+Q_{\mathrm{f}}^{\prime}\left(\bar{Q}_{\mathrm{f}}^{\prime}\right)$-jet $+X$, which could give us information not only on the IC contribution but also on the IS in the proton.

At $\mathrm{NLO}$ in $\mathrm{QCD}, W / Z+Q_{f}$ diagrams, often more complicated than the ones presented in Figs. 24 and 25 must also be considered. As can be seen in Fig. 26, the heavy flavor jets in the final state of these diagrams come from a gluon splitting somewhere along the event chain, and does thus not feature any intrinsic quark contribution. If the cross-sections of these diagrams is large 
enough, the conclusions about the sensitivity of a measurement to intrinsic charm at the LHC will be affected. It is thus important to consider QCD NLO excess in the $c$-jet $p_{\mathrm{T}}$ spectrum due to $\mathrm{IC}$ is of $\sim 5 \%$ for $p_{\mathrm{T}}$ of $50 \mathrm{GeV} / \mathrm{c}$, and rises to about $220 \%$ for $p_{\mathrm{T}} \sim 300 \mathrm{GeV} / \mathrm{c}$. This effect can thus be observed at 
the LHC if the $c$-jet $p_{\mathrm{T}}$ differential cross-section in $Z+c$ events can be measured with sufficient precision.

In the case of the $W$ production in association with heavy flavor jets, the intrinsic charm contribution would be observed in a $W+b$-jet final state due to the change of flavor in the charged current. In MCFM, the NLO $W+b$ Feynman diagrams for which the LO part is depicted in Fig. 25, correspond to the MCFM processes 12 and 17 [86. They provide the contribution to $W+Q^{\prime}$ which is sensitive to IC. The $p_{\mathrm{T}}$ spectrum of the $b$-jet is presented, for the sum of these processes, in Fig. 28 (top), where one calculation (squares) has been obtained at NLO in QCD with the CTEQ66c PDF that includes an IC contribution (about $3.5 \%$ ), and the other calculation (triangles) uses the CTEQ66 PDF, which does not include IC. On the bottom panel of Fig. 28, the ratio of these two spectra (with and without an IC contribution to the PDF used in the $W+b$ production calculations) is presented. From this figure, one can see that the inclusion of the IC contribution to the PDF leads to an increase in the $b$-jet spectrum by a factor of about 1.9 at $p_{\mathrm{T}}>250 \mathrm{GeV} / \mathrm{c}$. This is comparable to what was observed in the $Z+c$ case of Fig. 27 .
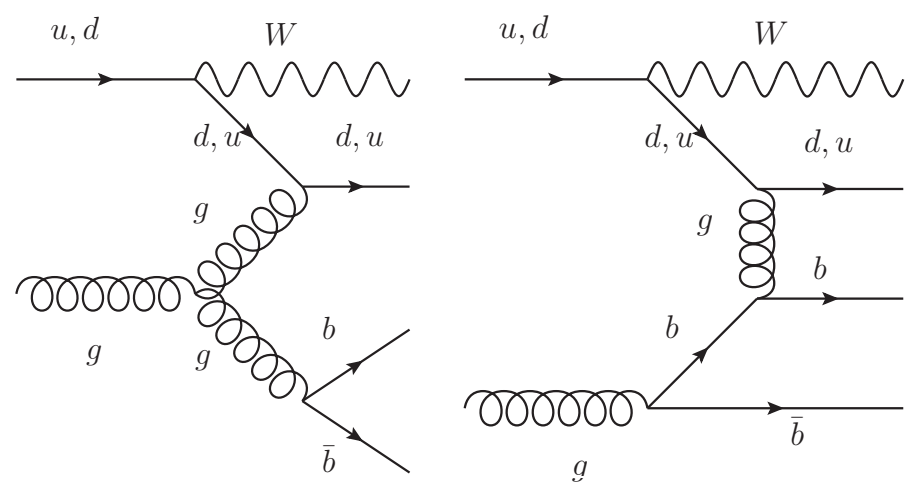

Figure 26: Some NLO Feynman diagrams for the process $Q_{f}\left(\bar{Q}_{f}\right) g \rightarrow W^{ \pm} Q_{f}^{\prime}\left(\bar{Q}_{f}^{\prime}\right)$, where $Q_{f}=c, b$ and $Q_{f}^{\prime}=b, c$ respectively. Left: gluon-splitting; Right: $t$-channel type of Wscattering with one gluon exchange in the intermediate state.

Similarly, the $W+c$ final state would be sensitive to intrinsic strange while 

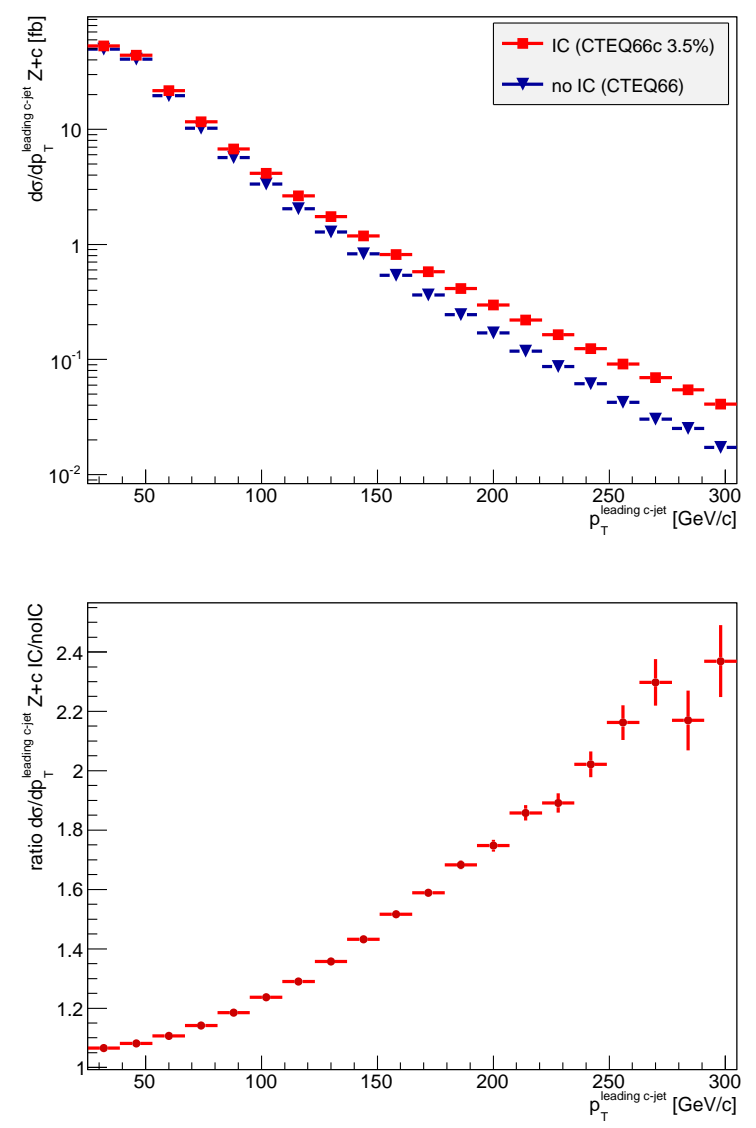

Figure 27: Comparison of the $p_{\mathrm{T}}$-spectra for the NLO $p p \rightarrow Z+c$ process 262 [86] obtained with PDF including an intrinsic charm component (CTEQ66c) and PDF having only an extrinsic component (CTEQ66) (top). Ratio of these two spectra (bottom).

the $Z+b$ final state would be sensitive to intrinsic bottom. These processes are however suboptimal for finding intrinsic quarks at the LHC. As mentioned above, the contribution of the IB to the PDF is suppressed by a factor of $\left(\frac{m_{\mathrm{c}}}{m_{\mathrm{b}}}\right)^{2}$ and is thus subdominant compared to intrinsic charm. The contribution of the intrinsic strangeness (IS) can be of the same order of magnitude as the IC according to [68, 81]. The $Q^{2}$ evolution for this component has however not been calculated up to now, and thus contains many unknowns. This is why this paper concentrate on the intrinsic charm component of the proton. 

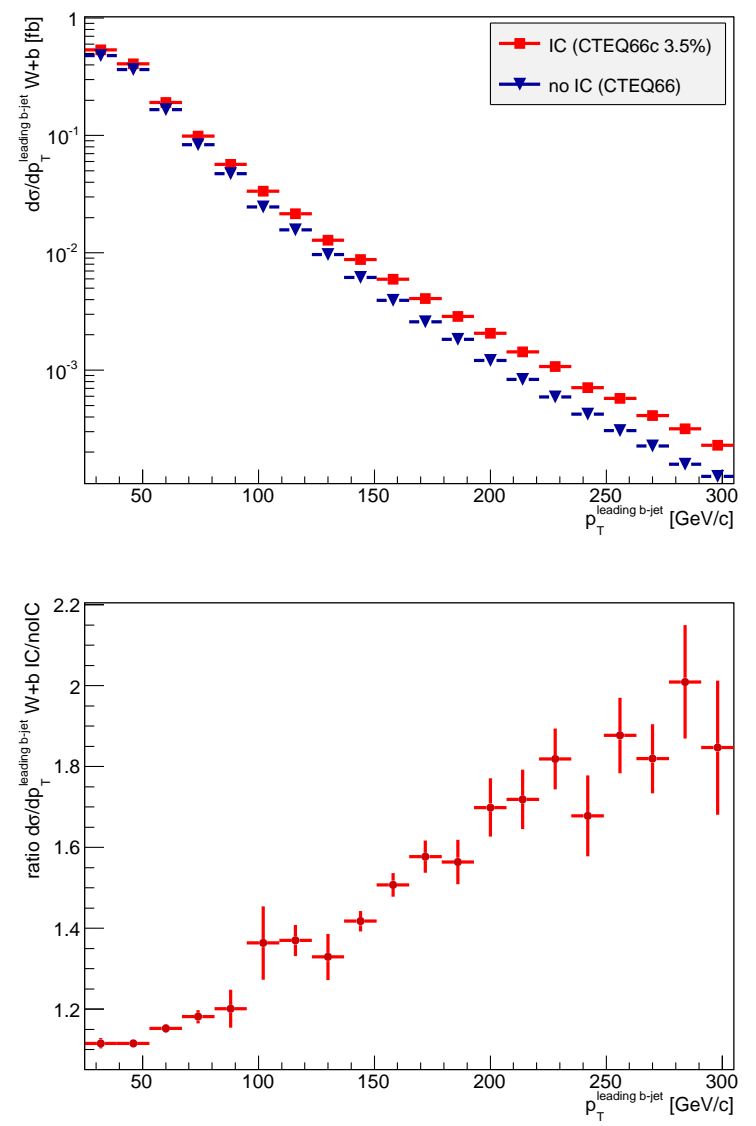

Figure 28: Comparison of the $p_{\mathrm{T}}$-spectra for the NLO $p p \rightarrow W+b$, processes $12+17$ [86] obtained with PDF including an intrinsic charm component (CTEQ66c) and PDF having only an extrinsic component (CTEQ66) (top). Ratio of these two spectra (bottom).

The above results of Figs. 27 and 28 seem a priori very encouraging regarding the capacity of the LHC to provide an observation of an intrinsic charm contribution to the PDFs in $W / Z+Q_{\mathrm{f}}$ events, but the real situation is unfortunately more complex than this. The $W$ boson plus one or more $b$-quark jets production, calculated at NLO in the 4-flavor scheme (4FNS), for which two of the diagrams are represented in Fig. 26 must also be included. These correspond to the MCFM processes number 401/406 and 402/407 [86]. Their total cross-section is about 50 times larger that the $W+b$ processes sensitive 
to IC. As a result, the total $W+b$ production is not sensitive to an intrinsic charm component of the PDF, as it was shown in [39, where the sum of all processes contributing to $W+b$ has been taken into account. Fortunately the $Z+c$ processes do not suffer from a similar large dilution of the intrinsic quark component because the $Q_{\mathrm{f}}+g \rightarrow Z+Q_{\mathrm{f}}$ processes, which are sensitive to IC, are not Cabibbo-suppressed.

Another difficulty lies in the experimental identification of heavy flavor jets in the way how to select them, for example, $Z+c$-jet events in a very large $Z$-jets sample. Algorithms typically disentangling heavy flavor jets from lightquark jets exploit the longer lifetime of heavy-quark hadrons that decay away from the primary vertex of the main process, but close enough to allow for a reconstruction of the tracks of the decay products of the heavy-flavor hadron ${ }_{940}$ in the inner part of the detector. Such algorithms are typically not capable of explicitly distinguishing $c$-jets from $b$-jets; only the efficiency for identifying the heavy flavor nature of the jet would differ between $c$-jets and $b$-jets. For example, one of the ATLAS heavy-flavor tagging algorithm (MV1) yields an efficiency of $85 \%$ for $b$-jet identification and $50 \%$ for $c$-jet (for a working point where the light flavor rejection is 10), see References in [39. As a result of such heavy flavor jet tagging algorithm, the selected $Z+Q$ final state will be a mixture of $Z+c$ and $Z+b$.

A priori, one would expect that $Z+b$ events are sensitive to intrinsic bottom and therefore act only as a small background to intrinsic charm studies, when the two processes cannot be experimentally distinguished. The situation is however more complicated than this. Because of sum rules, an intrinsic charm component would affect the total $b$-quark contribution to the proton, and the $Z+b$-jet final state therefore becomes sensitive to intrinsic charm as well. As was shown in [39], this contribution is in the opposite direction of the intrinsic charm effect on $Z+c$ processes presented in Fig. 27. In addition, the heavy flavor tagging efficiency is lower for $c$-jets than it is for $b$-jets, therefore increasing the weight of the negative $Z+b$ contribution to the total $Z$ plus heavy flavor tagged jets signal. The question is thus: are $Z+Q$-jet events still sensitive to intrinsic 
charm?

To avoid these difficulties in [39] the following idea to search the IC signal in the $Z / W$ boson production accompanied by heavy flavor jets was suggested. The new idea is to use the ratio of the leading heavy flavor spectra in inclusive heavy flavor $Z+Q$ to $W+Q$ events to verify the predictions about an IC contribution to the proton. The background related to the $Z / W$ production with association of light jets is included, which does not change the main conclusion on the IC signal in that ratio. We would like to stress also that studying the ratios discussed above we avoid many uncertainties related to a background due to the light jet production, rescaling in QCD and many others because they are almost cancelled. Such measurements can already be made with ATLAS and CMS available data.

To verify this, the ratio of the $p_{\mathrm{T}}$ spectra of the leading heavy flavor jet ( $b$, $c)$ produced in $Z b+Z c$ and $W b+W c+W b j$ processes has been to the proton. The of the calculation is presented in Fig. 29. As can be seen in this figure, the sensitivity to IC signal observed in $Z+Q$ is maintained in the ratio, which can amount to about $160 \%$ of the extrinsic only contribution at $p_{\mathrm{T}}$ of about $270-300 \mathrm{GeV} / \mathrm{c}$. This ratio measurement would, at least partially, cancel a number of large experimental systematic uncertainties, especially since in our proposal, $V+c$-jets and $V+b$-jets are both considered as signal and not treated as a background with respect to the other. This would allow for a clear signal at the LHC, if the IC contribution is sufficiently high (here we considered a $3.5 \%$ contribution). In the case where no excess is observed, limits on the IC contribution to the proton can be obtained from such measurement. Note that ratio predictions obtained with MCFM 39] would agree with predictions that include a parton shower and a modeling of the hadronization, because such 985 effects cancel in the ratio for jets above $\sim 100 \mathrm{GeV} / \mathrm{c}$.

As discussed above, a high $p_{\mathrm{T}}$ and relatively high rapidity heavy-flavor jet enhances the probability to have a heavy flavor quark in the initial state with a high- $x$ fraction, ensuring that the effect of intrinsic quarks on the cross-section is more prominent. This is the reason why we proposed to measure the ratio of $Z+$ 

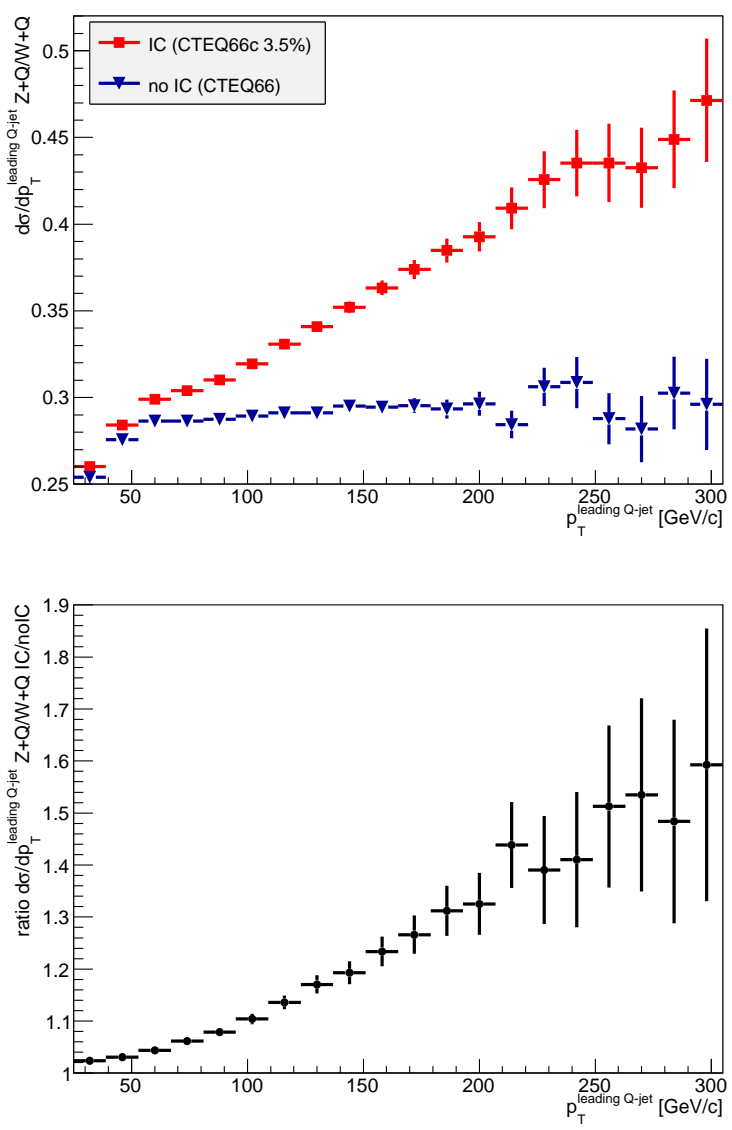

Figure 29: Comparison of the ratio of the $p_{\mathrm{T}}$-spectra for the $Z+Q$ to $W+Q$ NLO processes obtained with PDF including an intrinsic charm component (CTEQ66c) and PDF having only an extrinsic component (CTEQ66). Heavy flavor jet tagging efficiencies have been applied to the $c$-jets and the $b$-jets (top). Ratio of these two ratios of spectra (bottom).

$Q$ to $W+Q$ differential cross-sections as a function of the transverse momentum of the leading heavy-flavor jet measured within a specific rapidity interval. As indicated by Eq. 20, a large- $x$ heavy flavor quark will in general be achieved by a high-value of the Feynman variable $x_{\mathrm{F}}^{\mathrm{V}}$ of the final state vector boson $V$ recoiling to the hadronic system. While such variable cannot be reconstructed at the detector level in $W+Q$ events because of the presence of an undetectable neutrino in the final state, it is possible to construct a quantity highly correlated 
to such Feynman variable by using the leading heavy-flavor jet in the final state, rather than the vector boson. We therefore propose to investigate the sensitivity to IC of the ratio of the $Z+Q$ to $W+Q$ differential cross-sections as a function of the pseudo-Feynman variable of the leading heavy-flavor jet defined as:

$$
x_{\mathrm{F}}^{\mathrm{Q}}=\frac{2 p_{\mathrm{T}}^{\text {Lead } Q \text {-jet }}}{\sqrt{s}} \sinh \left(\eta_{Q}\right)
$$

where $p_{\mathrm{T}}^{\text {Lead } Q \text {-jet }}$ is the transverse momentum of the leading heavy flavor jet in the final state and $\eta_{Q}$ is the pseudo-rapidity of this jet.

First, the sensitivity of $Z+Q$ events to intrinsic charm is presented in the top panel of Fig. 30 as a function of this pseudo-Feynman variable of the leading heavy-flavor jet. The $x_{\mathrm{F}}^{\mathrm{Q}}$-spectrum has been obtained from the total NLO $p p \rightarrow Z+b(\bar{b})$ plus $p p \rightarrow Z+c(\bar{c})$ contributions calculated with MCFM (processes 261, 262 [86]) for collisions at $\sqrt{s}=8 \mathrm{TeV} / \mathrm{c}$. The distribution displayed with red square has been obtained using the CTEQ66c PDF distribution displayed with red square has been obtained using the CTEQ66c PDF that includes an intrinsic charm component, while the distribution displayed as blue inverted triangles have been obtained with the CTEQ66 PDF that only contains an extrinsic charm component. In the bottom panel the ratio of the two spectra is shown.

Fig. 31 presents the sensitivity to an intrinsic charm component to the PDF for the ratio of $Z+Q$ to $W+Q$ events as a function of the pseudo-Feynman variable $x_{\mathrm{F}}^{\mathrm{Q}}$. In this figure, heavy flavor tagging has been applied to both $Z+Q$ and $W+Q$ processes. An IC contribution of $3.5 \%$ yields a change by a factor of 2 to 4 in the $Z+Q$ to $W+Q$ cross-section ratio at $x_{\mathrm{F}}^{\mathrm{Q}} \simeq 0.3-0.4$ compared to the calculation where the PDF do not include any IC component. The number of events in that kinematic region runs from about a few hundred up to a few thousand events, for both $Z+Q$ and $W+Q$ processes. This results in a reduced statistical uncertainty on the $Z+Q$ to $W+Q$ ratio compared to the proposed ratio measured as a function of the transverse momentum of the leading heavy flavor jet in the phase space region discussed above. Because 
correspond to significantly different distributions in shape, both sensitive to an intrinsic charm contribution to the proton, but with different sensitivity to the various systematic uncertainties, we thus have here two complementary ratio observables to be measured at the LHC in order to observed an IC contribution to the proton, or determine an upper limit on it.
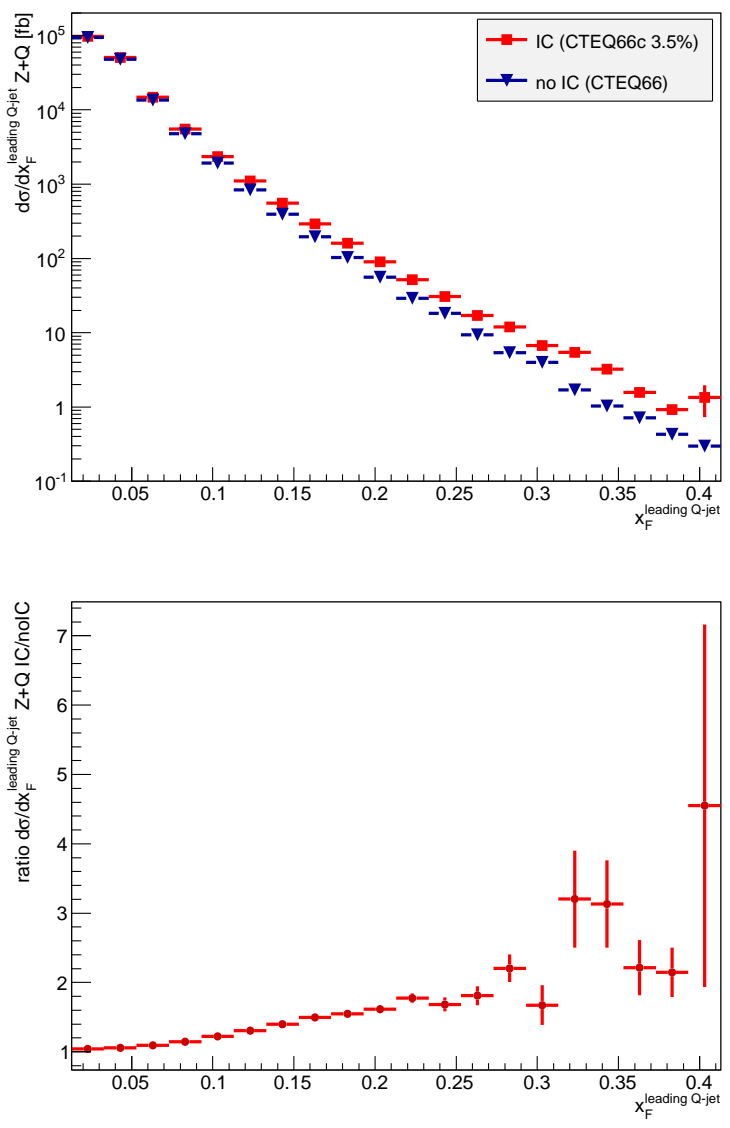

Figure 30: Comparison of the $x_{\mathrm{F}}^{\mathrm{Q}}$-spectra for the total NLO $p p \rightarrow Z+b(\bar{b})$ process plus $p p \rightarrow Z+c(\bar{c})$ (processes 261, 262 [86]) obtained with PDF including an intrinsic charm component (CTEQ66c) and PDF having only an extrinsic component (CTEQ66) (top). Ratio of these two spectra (bottom).

As discussed above, the leading heavy flavor jet transverse momentum and rapidity distributions are similar for $b$-jet and $c$-jet in $Z+Q$ and $W+Q$ events. 

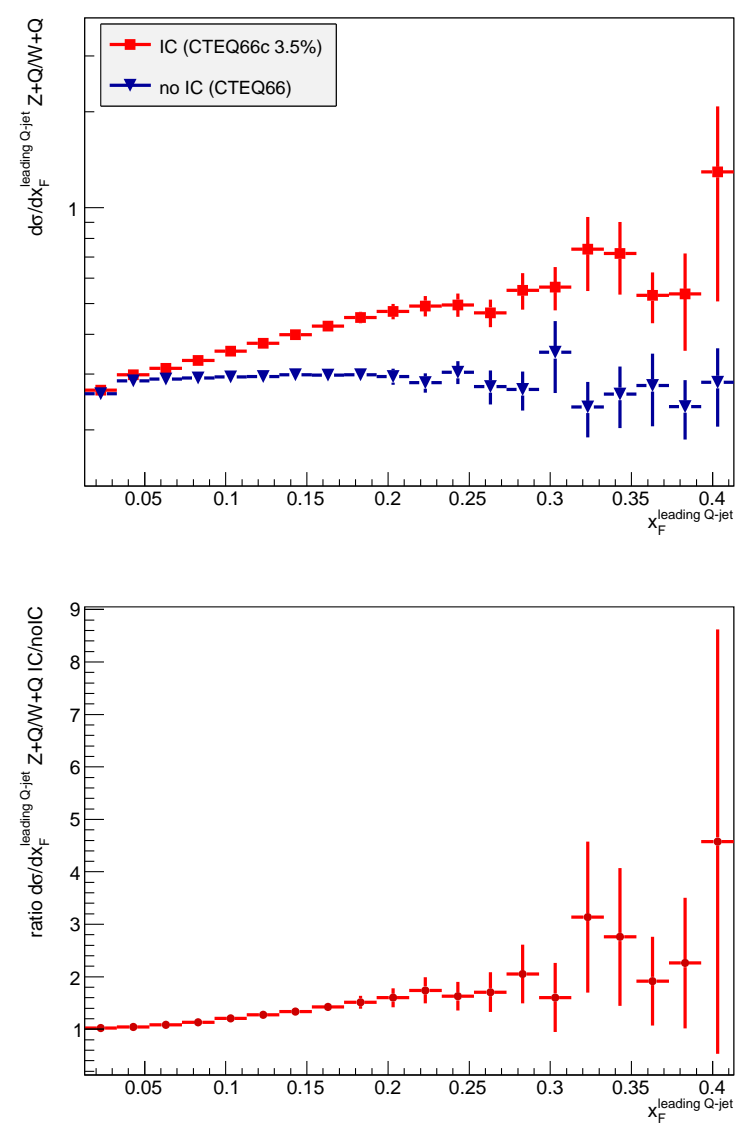

Figure 31: Comparison of the ratio of the $x_{\mathrm{F}}^{\mathrm{Q}}$-spectra for the $Z+Q$ to $W+Q$ NLO processes obtained with PDF including an intrinsic charm component (CTEQ66c) and PDF having only an extrinsic component (CTEQ66) (top). Ratio of these two ratios of spectra (bottom).

As a consequence, the experimental uncertainties on $Q$-jet energy measurements and heavy flavor tagging efficiencies will get significantly reduced in the ratio measurements proposed above. The Feynman diagrams contributing to $Z+Q$ and $W+Q$ processes are however quite different. It is therefore important to verify that a similar cancellation of the theory uncertainty also occurs in this ratio, therefore not impeding the conclusion about IC that can be obtained with such ratio. The dominant theoretical systematic uncertainty on a NLO cross- 
section calculation obtained at fixed order in perturbative QCD comes, by far, from the uncertainty introduced by the choice of renormalization $\left(\mu_{\mathrm{R}}\right)$ and factorization $\left(\mu_{\mathrm{F}}\right)$ scales in the calculation. In the current calculations performed with MCFM [86, the central predictions were obtained with a dynamic scale $\mu_{\mathrm{R}}=\mu_{\mathrm{F}}=H_{\mathrm{T}}$, where $H_{\mathrm{T}}$ is the scalar sum of the transverse momentum of

sensitivity of the calculations to this choice of scale, cross-sections have been calculated with two other choices of scale, $H_{\mathrm{T}} \cdot 2$ and $H_{\mathrm{T}} / 2$, and results compared to the nominal predictions.

It was shown in 39 that the choice of scale made in both predictions is the same, therefore leaving a ratio of predictions with IC to predictions without IC independent of the choice of scale.

\section{Conclusion}

The quark and gluon distribution functions of the proton encode the colorconfining dynamics of QCD. These probability distributions are directly related to the frame-independent light-front wavefunctions, the eigensolutions of the QCD Hamiltonian. Because of QCD factorization, the PDFs allow the computation of the cross sections for virtually all high energy collision processes studied at the LHC. However, the heavy quark contribution to the proton PDFs is still a major uncertainty. As we have discussed, QCD predicts two sources of heavy quarks to the constituent structure of the light hadrons - the standard small-x extrinsic contribution at from gluon splitting $g \rightarrow Q \bar{Q}-$ plus the intrinsic contribution at large $x$ which arises from diagrams where the $Q \bar{Q}$ pair is connected by two or more gluons to the valence quarks. The maximum configuration of such intrinsic contributions occur at minimal off-shellness; i.e., when all of the quarks in the $|u u d Q \bar{Q}\rangle$ Fock state are at rest in the proton's rest frame, corresponding to equal rapidity $y_{i}$ in the moving proton, and thus where the momentum fraction carried by each quark is proportional to its transverse mass: $x_{i} \propto \sqrt{m_{\mathrm{T} i}^{2}+\vec{k}_{\mathrm{T} i}^{2}}$. 
The hypothesis of intrinsic quark components in the proton at high $x$ was

orinally suggested in [6] was motivated possible explanation of the large crosssection for the forward open charm production in $p p$ collision at ISR energies [7[10. However, the accuracy of the experimental data on the open charm production at large $x$ does not provide precise constraints on the intrinsic heavy quark probability. We have discussed a number of experiments such as as deep inelastic scattering measurements of $c(c, Q)$ at the EMC and HERA; soft processes of the open charm or strangeness production and even the Higgs boson production in $p p$ collisions at LHC. We have also shown that the inclusive production of open charm or strangeness at high transverse momentum can serve as a tool for the search for the heavy quark $Q \bar{Q}$ Fock states in the nucleon. The IC or IS signal can be visible in the $p_{\mathrm{T}}$-spectrum of $D$-mesons or $K$-mesons produced, for example, in $p p$ collisions at their large rapidities $y$ and transverse momenta $p_{\mathrm{T}}$. It can be verified at CERN experiments such as the LHC and NA61.

The semi-inclusive production of prompt photons or gauge vector bosons in association with heavy flavor jets $c$ or $b$ provides an ideal method for verifying the IC contribution in the proton PDF. The increase of $p_{\mathrm{T}}$ spectrum of these hadrons or jets produced at large $p_{\mathrm{T}}$ and the forward rapidity region of ATLAS or CMS $(1.5<|y|<2.4)$ due to the IC enhancement in the PDF is predicted. We also have found observables very sensitive to the non-zero intrinsic charm contribution to the proton density. They are the $\gamma / Z+b$ production. These ratios should be decreasing in the absence of the IC contribution to the PDF and they should be flat or increasing if the IC is included, when $p_{\mathrm{T}}$ grows. This prediction, can also verify the IC hypothesis at LHC.

We argued that the ratio of the cross-sections $\gamma / Z+c$ and $\gamma / Z+b$ integrated over $p_{\mathrm{T}}>p_{\mathrm{T}}^{\min }$ with $p_{\mathrm{T}}^{\min } \geq 100 \mathrm{GeV} / \mathrm{c}$ can be used to determine the IC probability $w$ from the future LHC data because we calculated it as a function of $w$. The advantage of the proposed ratios is that the theoretical uncertainties are very small, while the uncertainties for the $p_{\mathrm{T}}$-spectra of photons or $Z$ bosons produced in association with the $c$ or $b$ jets are large. Therefore, the search for the IC signal by analyzing the ratio $\sigma(\gamma / Z+c) / \sigma(\gamma / Z+b)$ can be more 
promising.

We have also shown that because of dominance of gluon-splitting processes, the production of $W$-bosons accompanied by heavy flavor jets is not directly sensitive to intrinsic heavy quarks. However, we can take advantage of this fact to propose a promising new method which reduces the expected systematic uncertainties by comparing the differential cross-section for $W$ plus heavy flavor jets to that in $Z+Q$ events. The ratio of the leading heavy flavor spectra in inclusive heavy flavor $Z+Q$ to $W+Q$ events can thus be used to determine the intrinsic heavy quark contribution to the proton PDF. Such measurements can already be made with available data from ATLAS and CMS.

We have also discussed the fact that the existence of intrinsic heavy charm and bottom quarks in the proton wavefunction implies that the Higgs boson will be produced at high momentum fractions $x_{\mathrm{F}}>0.8$ in $p p \rightarrow H X$ collisions at the LHC, via the same mechanism that produces quarkonium states such as $p p \rightarrow J / \psi X$ at high $x_{\mathrm{F}}$. In addition, we have noted that fixed target experiments 1115 at the LHC such as the AFTER facility and the SMOG nuclear target at LHCb can materialize the intrinsic heavy quark Fock states in a novel way. Since the momentum distributions of the intrinsic heavy quarks in the $|u u d Q \bar{Q}\rangle$ Fock state are maximal when all of the constituents have the same rapidity, the collision of the LHC proton beam with nucleons in the nuclear target will lead to the production of heavy hadrons such as the $\Lambda_{\mathrm{b}}$ and exotic heavy quark states, such as tetraquarks and pentaquarks, at small rapidities relative to the rapidity of the target. Each of these novel processes will illuminate one of the most interesting features of QCD bound state dynamics - intrinsic heavy quarks.

\section{Acknowledgements}

We thank A. Glazov for extremely helpful discussions and recommendations for the predictions on the search for the possible intrinsic heavy flavor components in $p p$ collisions at high energies. We are also grateful to P-H. Beauchmin, T. Dado, M. Demichev, N. Husseynov, A.V. Lipatov, T. Stavreva, Yu. Stepan- 
neko and M. Stockton for very productive collaboration, H. Jung, R. Keys, and M. Williams for very helpful discussions. This research was supported in part by the Department of Energy contract DE-AC02-76SF00515 SLAC-PUB16844.

\section{References}

[1] V. N. Gribov, L. N. Lipatov, Sov. J. Nucl. Phys. 15 (1972) 438-450. [Yad. Fiz.15,781(1972)].

[2] G. Altarelli, G. Parisi, Nucl. Phys. B126 (1977) 298. doi 10.1016/ 0550-3213(77)90384-4.

[3] Y. L. Dokshitzer, Sov. Phys. JETP 46 (1977) 641-653. [Zh. Eksp. Teor. Fiz.73,1216(1977)].

[4] S. J. Brodsky, J. C. Collins, S. D. Ellis, J. F. Gunion, A. H. Mueller, in: ELECTROWEAK SYMMETRY BREAKING. PROCEEDINGS, WORKSHOP, BERKELEY, USA, JUNE 3-22, 1984. URL:http://www-public.slac.stanford.edu/sciDoc/docMeta. aspx?slacPubNumber=SLAC-PUB-15471.

[5] M. Franz, M. V. Polyakov, K. Goeke, Phys. Rev. D62 (2000) 074024. doi 10.1103/PhysRevD.62.074024, arXiv:hep-ph/0002240.

[6] S. J. Brodsky, P. Hoyer, C. Peterson, N. Sakai, Phys. Lett. B93 (1980) 451-455. doi:10.1016/0370-2693(80)90364-0.

1150

[7] D. Drijard, et al. (CERN-College de France-Heidelberg-Karlsruhe), Phys. Lett. B81 (1979) 250-254. doi:10.1016/0370-2693(79)90535-5.

q [8] K. L. Giboni, et al., Phys. Lett. B85 (1979) 437-442. doi 10.1016/ 0370-2693(79)91291-7. 
[9] W. S. Lockman, T. Meyer, J. Rander, P. Schlein, R. Webb, S. Er1155 \\an, J. Zsembery, Phys. Lett. B85 (1979) 443-446. doi 10.1016/ 0370-2693(79)91292-9.

[ [10] D. Drijard, et al. (ACCDHW), Phys. Lett. B85 (1979) 452-457. doi 10. 1016/0370-2693(79)91294-2.

[11] R. Laha, S. J. Brodsky (2016). arXiv:1607.08240

1160 [12] A. Aktas, et al. (H1), Eur. Phys. J. C45 (2006) 23-33. doi:10.1140/epjc/ s2005-02415-6. arXiv:hep-ex/0507081.

q [13] S. J. Brodsky, C. Peterson, N. Sakai, Phys. Rev. D23 (1981) 2745. doi 10. 1103/PhysRevD.23.2745.

q [14] S. J. Brodsky, Few Body Syst. 36 (2005) 35-52. doi 10.1007/ 1165 s00601-004-0077-8 arXiv:hep-ph/0411056.

[15] S. J. Brodsky, A. Kusina, F. Lyonnet, I. Schienbein, H. Spiesberger,

1. R. Vogt, Adv. High Energy Phys. 2015 (2015) 231547. doi:10.1155/2015/ 231547, arXiv:1504.06287

[ [16] J. Pumplin, Phys. Rev. D73 (2006) 114015. doi:10.1103/PhysRevD.73. $1170 \quad 114015$ arXiv:hep-ph/0508184.

[17] J. J. Aubert, et al. (European Muon), Nucl. Phys. B213 (1983) 31-64. doi $10.1016 / 0550-3213(83) 90174-8$

[18] R. D. Ball, V. Bertone, M. Bonvini, S. Carrazza, S. Forte, A. Guffanti, N. P. Hartland, J. Rojo, L. Rottoli (NNPDF) (2016). arXiv:1605.06515

1175 [ [19] R. D. Ball, et al. (NNPDF), JHEP 04 (2015) 040. doi 10.1007/ JHEP04(2015)040, arXiv:1410.8849.

[20] S. Dulat, T.-J. Hou, J. Gao, M. Guzzi, J. Huston, P. Nadolsky, J. Pumplin, C. Schmidt, D. Stump, C. P. Yuan, Phys. Rev. D93 (2016) 033006. doi:10. 1103/PhysRevD.93.033006. arXiv:1506.07443. 
1180 [21] M. Adamovich, et al. (WA82), Phys. Lett. B305 (1993) 402-406. doi: 10. 1016/0370-2693(93) 91074-W

[ [22] G. A. Alves, et al. (E769), Phys. Rev. Lett. 72 (1994) 812-815. doi-10. 1103/PhysRevLett.72.812, [Erratum: Phys. Rev. Lett.72,1946(1994)].

a [23] E. M. Aitala, et al. (E791), Phys. Lett. B371 (1996) 157-162. doi 10. 1185 1016/0370-2693(96)00093-7. arXiv:hep-ex/9601001.

q [24] A. B. Kaidalov, Phys. Lett. B116 (1982) 459-463. doi 10.1016/ 0370-2693(82)90168-X.

[25] A. Capella, U. Sukhatme, C.-I. Tan, J. Tran Thanh Van, Phys. Rept. 236 (1994) 225-329. doi 10.1016/0370-1573(94)90064-7.

${ }_{1190}^{\text {_ }}$ [26] A. B. Kaidalov, O. I. Piskunova, Z. Phys. C30 (1986) 145. doi 10.1007/ BF01560688

[ [27] Yu. M. Shabelski, Surveys High Energ. Phys. 9 (1995) 1-88. doi 10.1080/ 01422419508225681

[28] G. I. Lykasov, G. H. Arakelian, M. N. Sergeenko, Phys. Part. Nucl. 30 (1999) 343-368. doi:10.1134/1.953111, [Fiz. Elem. Chast. Atom. Yadra30,817(1999)].

q [29] S. Barlag, et al. (ACCMOR), Phys. Lett. B247 (1990) 113-120. doi 10. 1016/0370-2693(90)91058-J.

a [30] G. Bari, et al., Nuovo Cim. A104 (1991) 571-599. doi 10.1007/ $1200 \quad$ BF02813593

q [31] V. M. Abazov, et al. (D0), Phys. Rev. Lett. 102 (2009) 192002. doi 10. 1103/PhysRevLett.102.192002, arXiv:0901.0739.

[ [32] V. M. Abazov, et al. (D0), Phys. Lett. B714 (2012) 32-39. doi 10.1016/ j.physletb.2012.06.056. arXiv:1203.5865. 
1205 [33] V. M. Abazov, et al. (D0), Phys. Lett. B719 (2013) 354-361. doi 10.1016/ j.physletb.2013.01.033 arXiv:1210.5033.

[ [34] T. Aaltonen, et al. (CDF), Phys. Rev. D81 (2010) 052006. doi 10.1103/ PhysRevD.81.052006. arXiv:0912.3453.

q [35] T. Aaltonen, et al. (CDF), Phys. Rev. Lett. 111 (2013) 042003. doi 10. 1103/PhysRevLett.111.042003. arXiv:1303.6136.

[36] M. V. Polyakov, A. Schafer, O. V. Teryaev, Phys. Rev. D60 (1999) 051502. doi 10.1103/PhysRevD.60.051502. arXiv:hep-ph/9812393.

q [37] T. P. Stavreva, J. F. Owens, Phys. Rev. D79 (2009) 054017. doi 10.1103/ PhysRevD.79.054017, arXiv:0901.3791.

${ }_{1215}$ [38] V. A. Bednyakov, M. A. Demichev, G. I. Lykasov, T. Stavreva, M. Stock口 ton, Phys. Lett. B728 (2014) 602-606. doi 10.1016/j.physletb.2013. 12.031. arXiv: 1305.3548

[39] P.-H. Beauchemin, V. A. Bednyakov, G. I. Lykasov, Yu. Yu. Stepanenko, Phys. Rev. D92 (2015) 034014. doi 10.1103/PhysRevD.92.034014. arXiv:1410.2616.

[40] A. V. Lipatov, G. I. Lykasov, Yu. Yu. Stepanenko, V. A. Bednyakov, Phys. Rev. D94 (2016) 053011. doi:10.1103/PhysRevD.94. 053011, arXiv:1606.04882

[41] S. J. Brodsky, B. Kopeliovich, I. Schmidt, J. Soffer, Phys. Rev. D73 (2006) 113005. doi:10.1103/PhysRevD.73.113005. arXiv:hep-ph/0603238.

[42] S. J. Brodsky, A. S. Goldhaber, B. Z. Kopeliovich, I. Schmidt, Nucl. Phys. B807 (2009) 334-347. doi 10.1016/j.nuclphysb.2008.09.014 arXiv:0707.4658.

[43] S. J. Brodsky, F. Fleuret, C. Hadjidakis, J. P. Lansberg, Phys. 1230 Rept. 522 (2013) 239-255. doi 10.1016/j.physrep.2012.10.001 arXiv: 1202.6585 . 
[ [44] J. W. Negele, et al., Nucl. Phys. Proc. Suppl. 128 (2004) 170-178. doi 10. 1016/S0920-5632(03)02474-5, arXiv:hep-lat/0404005, [,170(2004)].

q [45] W. Schroers, Nucl. Phys. A755 (2005) 333-336. doi 10.1016/j. 1235 nuclphysa.2005.03.034. arXiv:hep-ph/0501156.

[46] J. Pumplin, D. R. Stump, J. Huston, H. L. Lai, P. M. Nadolsky, W. K. Tung, JHEP 07 (2002) 012. doi:10.1088/1126-6708/2002/07/012. arXiv:hep-ph/0201195.

[47] D. Stump, J. Huston, J. Pumplin, W.-K. Tung, H. L. Lai, S. Kuhlmann, J. F. Owens, JHEP 10 (2003) 046. doi:10.1088/1126-6708/2003/10/046 arXiv:hep-ph/0303013.

[48] R. S. Thorne, A. D. Martin, W. J. Stirling, R. G. Roberts, in: Deep inelastic scattering. Proceedings, 12th International Workshop, DIS 2004, Strbske Pleso, Slovakia, April 14-18, 2004. Vol. $1+2$, pp.

1245 4 427-432. URL: http://wWW.saske.sk/dis04/proceedings/A/thorne. ps.gz, arXiv:hep-ph/0407311.

q [49] S. J. Brodsky, B.-Q. Ma, Phys. Lett. B381 (1996) 317-324. doi 10.1016/ 0370-2693(96)00597-7. arXiv: hep-ph/9604393.

q [50] J. Pumplin, H. L. Lai, W. K. Tung, Phys. Rev. D75 (2007) 054029. doi 10. 1103/PhysRevD .75.054029, arXiv:hep-ph/0701220.

[ [51] J. F. Donoghue, E. Golowich, Phys. Rev. D15 (1977) 3421. doi 10.1103/ PhysRevD.15.3421.

[52] S. Paiva, M. Nielsen, F. S. Navarra, F. O. Duraes, L. L. Barz, Mod. Phys. Lett. A13 (1998) 2715-2724. doi:10.1142/S0217732398002886

1255 arXiv:hep-ph/9610310.

[ [53] W. Melnitchouk, A. W. Thomas, Phys. Lett. B414 (1997) 134-139. doi 10. 1016/S0370-2693(97)01150-7. arXiv: hep-ph/9707387. 
[54] F. M. Steffens, W. Melnitchouk, A. W. Thomas, Eur. Phys. J. C11 (1999) 673-683. doi $10.1007 /$ s100529900189,10.1007/s100520050663 arXiv:hep-ph/9903441.

[55] T. J. Hobbs, J. T. Londergan, W. Melnitchouk, Phys. Rev. D89 (2014) 074008. doi 10.1103/PhysRevD.89.074008, arXiv:1311.1578.

[56] P. M. Nadolsky, H.-L. Lai, Q.-H. Cao, J. Huston, J. Pumplin, D. Stump, W.-K. Tung, C. P. Yuan, Phys. Rev. D78 (2008) 013004. doi 10.1103/ PhysRevD .78.013004, arXiv:0802.0007.

q [57] S. J. Brodsky, S. Gardner, Phys. Rev. Lett. 116 (2016) 019101. doi:10. 1103/PhysRevLett.116.019101. arXiv:1504.00969.

n [58] R. Ammar, et al. (LEBC-MPS), Phys. Lett. B183 (1987) 110. doi 10. 1016/0370-2693(87)91427-4, [Erratum: Phys. Lett.B192,478(1987)].

${ }_{1270}$ [59] J. Russ, et al. (SELEX), in: High-energy physics. Proceedings, 29th International Conference, ICHEP'98, Vancouver, Canada, July 23-29, 1998. Vol. 1, 2, pp. 1259-1262. URL: http://alice.cern.ch/format/ showfull?sysnb=0300703, arXiv: hep-ex/9812031.

[ [60] S. J. Brodsky, Nucl. Part. Phys. Proc. 258-259 (2015) 23-30. doi 10.1016/ $1275 \quad$ j.nuclphysbps.2015.01.007. arXiv:1410.0404

[ [61] J. Blmlein, Phys. Lett. B753 (2016) 619-621. doi:10.1016/j.physletb. 2015.12.068, arXiv:1511.00229

[62] S. Catani, D. de Florian, G. Rodrigo, W. Vogelsang, Phys. Rev. Lett. 93 (2004) 152003. doi 10.1103/PhysRevLett.93.152003 arXiv:hep-ph/0404240.

口 [63] F. G. Garcia, et al. (SELEX), Phys. Lett. B528 (2002) 49-57. doi 10 . 1016/S0370-2693(01)01484-8, arXiv:hep-ex/0109017. 
[64] S. Rostami, A. Khorramian, A. Aleedaneshvar, M. Goharipour, J. Phys. G43 (2016) 055001. doi:10.1088/0954-3899/43/5/055001 arXiv:1510.08421

[65] S. Catani, M. Ciafaloni, F. Hautmann, Nucl. Phys. B366 (1991) 135-188. doi $10.1016 / 0550-3213(91) 90055-3$

[66] G. I. Lykasov, V. A. Bednyakov, A. F. Pikelner, N. I. Zimine, Europhys. Lett. 99 (2012) 21002. doi 10.1209/0295-5075/99/21002 arXiv:1205.1131.

q [67] V. P. Goncalves, F. S. Navarra, Nucl. Phys. A842 (2010) 59-71. doi 10. 1016/j.nuclphysa.2010.04.004. arXiv:0805.0810.

[68] J.-C. Peng, W.-C. Chang, PoS QNP2012 (2012) 012. arXiv:1207.2193.

[69] A. Airapetian, et al. (HERMES), Phys. Lett. B666 (2008) 446-450. doi $10.1016 /$ j.physletb.2008.07.090 arXiv:0803.2993.

[70] V. A. Litvine, A. K. Likhoded, Phys. Atom. Nucl. 62 (1999) 679-692. [Yad. Fiz.62,728(1999)].

a [71] A. V. Lipatov, M. A. Malyshev, N. P. Zotov, JHEP 05 (2012) 104. doi 10. 1007/JHEP05(2012) 104, arXiv:1204.3828.

${ }_{1300}^{\text {口 }}$ [72] A. V. Lipatov, N. P. Zotov, J. Phys. G34 (2007) 219. doi 10.1088/ 0954-3899/34/2/005, arXiv:hep-ph/0507243.

q [73] R. Vogt, Prog. Part. Nucl. Phys. 45 (2000) S105-S169. doi 10.1016/ S0146-6410(00)90012-7, arXiv: hep-ph/0011298.

[74] F. S. Navarra, M. Nielsen, C. A. A. Nunes, M. Teixeira, Phys. Rev. D54 (1996) 842-846. doi 10.1103/PhysRevD.54.842 arXiv: hep-ph/9504388.

[75] J. C. Collins, D. E. Soper, G. F. Sterman, Adv. Ser. Direct. High Energy Phys. 5 (1989) 1-91. doi:10.1142/9789814503266_0001 arXiv:hep-ph/0409313. 
n [83] M. L. Mangano, Phys. Usp. 53 (2010) 109-132. doi:10.3367/UFNe.0180. 201002a.0113, [Usp. Fiz. Nauk180,113(2010)].

[84] L. Rottoli, in: Proceedings, 24th International Workshop on Deep-

[ [85] R. Aaij, et al. (LHCb), JINST 9 (2014) P12005. doi 10.1088/1748-0221/ 9/12/P12005, arXiv:1410.0149.

q [86] J. M. Campbell, R. K. Ellis, Phys. Rev. D65 (2002) 113007. doi 10.1103/ 1335

[76] R. P. Feynman, R. D. Field, G. C. Fox, Phys. Rev. D18 (1978) 3320. doi $10.1103 /$ PhysRevD. 18.3320 .

[77] V. A. Bednyakov, A. A. Grinyuk, G. I. Lykasov, M. Poghosyan, Int. J. Mod. Phys. A27 (2012) 1250042. doi 10.1142/S0217751X1250042X arXiv:1104.0532.

[78] B. A. Kniehl, G. Kramer, I. Schienbein, H. Spiesberger, Eur. Phys. J. C72 (2012) 2082. doi 10.1140/epjc/s10052-012-2082-2 arXiv:1202.0439

[79] A. Airapetian, et al. (HERMES), Phys. Rev. D89 (2014) 097101. doi 10. 1103/PhysRevD.89.097101, arXiv:1312.7028.

[80] D. de Florian, R. Sassot, M. Stratmann, Phys. Rev. D75 (2007) 114010. doi 10.1103/PhysRevD.75.114010, arXiv:hep-ph/0703242.

[81] G. I. Lykasov, I. V. Bednyakov, M. A. Demichev, Yu. Yu. Stepanenko, Nucl. Phys. Proc. Suppl. 245 (2013) 215-222. doi:10.1016/j. nuclphysbps .2013.10.042, arXiv:1309.3168.

[82] S. Albino, B. A. Kniehl, G. Kramer, Nucl. Phys. B803 (2008) 42-104. doi $10.1016 / j . n u c l p h y s b .2008 .05 .017$ arXiv:0803.2768. Inelastic Scattering and Related Subjects (DIS 2016). URL: https: //inspirehep.net/record/1473188/files/arXiv:1606.09289.pdf. arXiv:1606.09289.

\footnotetext{
PhysRevD.65.113007, arXiv:hep-ph/0202176.
} 
[87] E. M. Levin, M. G. Ryskin, Yu. M. Shabelski, A. G. Shuvaev, Sov. J. Nucl. Phys. 53 (1991) 657. [Yad. Fiz.53,1059(1991)].

[88] L. V. Gribov, E. M. Levin, M. G. Ryskin, Phys. Rept. 100 (1983) 1-150. doi $10.1016 / 0370-1573(83) 90022-4$

${ }_{1340}$ [89] E. A. Kuraev, L. N. Lipatov, V. S. Fadin, Sov. Phys. JETP 44 (1976) 443-450. [Zh. Eksp. Teor. Fiz.71,840(1976)].

[90] E. A. Kuraev, L. N. Lipatov, V. S. Fadin, Sov. Phys. JETP 45 (1977) 199-204. [Zh. Eksp. Teor. Fiz.72,377(1977)].

[91] I. I. Balitsky, L. N. Lipatov, Sov. J. Nucl. Phys. 28 (1978) 822-829. [Yad. Fiz.28,1597(1978)].

\ [92] B. Andersson, et al. (Small x), Eur. Phys. J. C25 (2002) 77-101. doi 10. 1007/s10052-002-0998-7, arXiv:hep-ph/0204115.

[ [93] J. R. Andersen, et al. (Small x), Eur. Phys. J. C35 (2004) 67-98. doi 10. 1140/epjc/s2004-01775-7. arXiv: hep-ph/0312333

${ }_{1350}^{\text {口 }}$ [94] J. R. Andersen, et al. (Small x), Eur. Phys. J. C48 (2006) 53-105. doi 10. 1140/epjc/s2006-02615-6. arXiv:hep-ph/0604189

[ [95] L. N. Lipatov, M. I. Vyazovsky, Nucl. Phys. B597 (2001) 399-409. doi 10. 1016/S0550-3213(00)00709-4. arXiv:hep-ph/0009340.

q [96] A. V. Bogdan, V. S. Fadin, Nucl. Phys. B740 (2006) 36-57. doi 10.1016/ 1355 j.nuclphysb.2006.01.033 arXiv:hep-ph/0601117.

q [97] M. Hentschinski, A. Sabio Vera, Phys. Rev. D85 (2012) 056006. doi 10. 1103/PhysRevD.85.056006. arXiv:1110.6741.

\[98] M. Hentschinski, Nucl. Phys. B859 (2012) 129-142. doi:10.1016/j. nuclphysb.2012.02.001, arXiv:1112.4509. 
[99] G. Chachamis, M. Hentschinski, J. D. Madrigal Martinez, A. Sabio Vera,

n Nucl. Phys. B861 (2012) 133-144. doi 10.1016/j.nuclphysb.2012.03. 015. arXiv:1202.0649,

[100] L. N. Lipatov, Nucl. Phys. B452 (1995) 369-400. doi 10.1016/ 0550-3213(95) 00390-E arXiv:hep-ph/9502308.

1365 [101] L. N. Lipatov, Phys. Rept. 286 (1997) 131-198. doi 10.1016/ S0370-1573(96)00045-2, arXiv:hep-ph/9610276.

[102] A. V. Lipatov, M. A. Malyshev (2016). arXiv:1606.02696.

[103] A. D. Martin, M. G. Ryskin, G. Watt, Eur. Phys. J. C66 (2010) 163-172. doi 10.1140/epjc/s10052-010-1242-5. arXiv:0909.5529.

1370 [104] K. A. Olive, et al. (Particle Data Group), Chin. Phys. C38 (2014) 090001. doi $10.1088 / 1674-1137 / 38 / 9 / 090001$. 